\title{
Assistive Robotic Micro-Rooms for Independent Living
}

\author{
Thomas Linner, Jörg Güttler, Thomas Bock, Christos Georgoulas* \\ Chair of Building Realization and Robotics, Technical University Munich, Germany \\ *Corresponding author. Tel.:+ 4989289 28650; fax:+49 8928922163 \\ E-mail address: christos.georgoulas@br2.ar.tum.de
}

\begin{abstract}
The demographic change situation in Germany, as well as in other leading industrial nations, leads to an overload of the family and institutionalized nursing care system structure. For this reason, novel solutions must be developed, in order to guarantee fully independent living of the elderly people, and an efficient assistance or nursing, in diverse living environments. The research project LISA has investigated the possibilities to embed mechatronic, assistive functions and services into compact wall "terminal” elements thereby enabling autonomous and independent living upon performing Activities of Daily Living (ADLs) by means of generated structured environments and robotic micro-rooms (RmRs). In this article, a classification of the approach in the broader context of research, as well as a statement of methodical and structured multi-phase development are described. The conceptual and development phases and the technical details using selected assistive functions of the entire system are presented. The article explains the methodical approach throughout the four phases of development and focuses on essential field trials with collected user feedback.
\end{abstract}

Keywords: Activities of Daily Living, Robotic Rooms, Service Robotics, Modularity, Ambient Assisted Living

\section{Introduction}

Due to natural aging processes, in a biological/medical and cognitive sense, it is more difficult for people to master ADLs on their own [1-4]. If someone loses the ability to self-maintain at home, care in nursing homes or by a family member is usually necessary. ADLs, refer to basic tasks of everyday life, such as eating, bathing, dressing, toileting, and transferring. When people are unable to perform these, they require assistance in order to cope, either from other human beings, or assistive devices, or both. Although persons of all ages may have problems performing the ADLs, prevalence rates are much higher for the elderly than for the nonelderly. Within the elderly population, ADL prevalence rates rise steeply with advancing age and are especially high for persons aged 85 and over [3]. However, the demographic change problem in Germany, but also present in other leading industrial nations such as Japan, Korea and China, leads to an overload for the family members and institutionalized nursing systems. At the same time, a reduction in 
financing for older people is observed, as well as in the economic productivity within Germany, as there are fewer employed productive individuals [5]. Furthermore, many elderly people live apart from their family (e.g. their children), usually for employment location related reasons. This is even more prevalent for elderly people living in rural areas. Various research projects are being carried out by the Chair for Building Realization and Robotics that deal with how to enable independent and dignified living for elderly people, by intelligent environment equipment which can be considered as novel approaches in the field of gerontechnology. Thus, for example in the research projects PASSAge [6] and GEWOS [7], the integration of assistive functions in mobility products and home furniture under complex, interdisciplinary research collaborations was investigated. In the research project LISA (Living Independently in South Tyrol Alto Adige), which is the focus of this article, the authors examined the possibility to integrate assistive functions in walls or wall components to generate structured environments, so-called RmRs, that can also be retrofitted into older buildings without requiring high costs, to enable independent and self-oriented living of elderly people. The economic potential of such a solution has been classified as very large by participating industrial partners concerning the middle- and long-term aspects, as it was noted that all currently emerging industrial global nations are experiencing the process of demographic change [8], while the trend towards individualization and self-centeredness in society progresses and therefore classic family assistance structures are being dismantled [9].

In section 1, a outline of the research need and research question followed by a classification of the approach in the broader context of research and the methodical and structured approach in the developing phases is made. In section 2, the concept of the overall system is described. In section 3 , the technical details of the selected assistive functions are presented. Section 4 describes the methodological approach in the four phases of development and is focused on gathering and integrating test-user feedback in several development cycles. Section 5 depicts how the system was designed in order to develop a higher-level product-service system. In section 6, the most important milestones and research results regarding the research project LISA are summarized. Finally, section 7 shows how obtained research results can be exploited and provides an outlook on the already initiated related research, which focuses on scaling the system in terms of assistive functions.

\subsection{Related Work and Research Question}

Some researchers have already proposed approaches to integrating mechatronics and robotic technologies into environments and to create robotized environments. Robotic Rooms [10], Wabot House [11] or Robot Town [12] have investigated the important basic approaches and foundations in this field of research. Robotic environments differ in regard to complexity, quality and multidimensionality of possible assistive functions relative to the classic "smart home" approach (the 
precise definition of the "smart home"-approach of assistive and robotic environments is discussed in detail in [13]). In [14], environments that are provided with a certain degree of intelligence complexity, autonomy and a plurality of distributed and embedded sensors or microprocessors are analyzed and referred to as "immobile robots". Since the 1980s, several research groups have created environments and prototype buildings for so-called smart buildings. Based on Ken Sakamura's T-Engine Hardware and a complementary operating system, the Tron House 1, 2 and 3 have been built [15]. The US AwareHome [16] and PlaceLab [17] follow a similar approach and MIT's House_n [18] even includes modular intelligent furniture that can be equipped with various sensor systems. Recently designed German prototypes of assistive homes, such as "Haus der Gegenwart" (house of presence) [19] and "Haus der Zukunft" (house of the future) [20], are exemplarily equipped with a variety of networked pervasive technologies integrated by modern design. Similar to our approach, Smart Buildings and Robotic Rooms try to integrate sensoractuator systems with architectonic elements. However, these approaches primarily integrate sensors, actuators and robots on an informational level. Furthermore, they are presenting implementations that are realized in a controlled experimental environment, and cannot be straightforwardly applied into a regular medium sized apartment to serve as an integrated assistive system for ADLs.

The multi-dimensionality of assistance, which is the ability to serve a variety of constraints simultaneously, can be created by robotic environments due to the expected multi-morbidity in ageing society which usually leads to complex, individual and multiple constraints [21]. Conceptual foundations for assistive, robotic rooms that are generated by compact room-in-space elements have already been set out by the authors in [22].

\subsection{Research Question and Method}

From the background described above it can be concluded, that the real-world utilization of assistive, robotic elements, i.e. robotic rooms for the purpose of ADL-assistance and care would provide a multitude of opportunities for future gerontechnology.Most previous approaches, however, are focused on the pure research aspect and not on an adaptation of concrete scenarios in collaboration with users and other determining factors such as the manufacturability of the system and its integration into an overarching value system.. Research has so far failed to address the question how basic research in robotic rooms could be translated into real world applications resulting in a lack of knowledge and guidelines. How could the idea of assistive, robotic rooms be translated into practical and user accepted approaches? How could those approaches add value to the life of elderly people, their relatives and caretakers? How could robotic elements be efficiently manufactured and integrated into exiting and newly constructed buildings? What product structures and business concepts would be reasonable and should therefore be considered as inputs for design 
and enginnering?

The LISA project attempts to address the existing lack of knowledge and guidelines by developing, testing and evaluating RmR systems according to standard residential configurations. In this project, the user is in the foreground. As elderly people are less receptive to new and more complex technology, it must be discreetly embedded in these RmRs. The control of the embedded intelligent systems is possible through intuitive gestures and voice commands which ensure that even elderly people can easily familiarize themselves with the operation [23, 24].

In the LISA project, a dedicated modular approach has been followed to form the basis for a cost efficient system development by the participating corporate partners. The approach pursues relatively recent modularization strategies in architecture and construction $[25,26]$, which are already connected, for example in Japan, with an automated production of buildings and building elements [27]. Such strategies, especially in the Ambient Assisted Living (AAL) field, have been implemented and investigated in [28]. Consequently, a comprehensive product architecture was created, physical-wise and information technology-wise, as well as a furniture-like wall element, (Figure 1), which creates the basis for the various functions of the RmR described in LISA: (i) new assistive functions can be "plugged in", (ii) remote access to i.e. vital signs data by a physician or care provider via internet, and (iii) the platform can be further expanded to be integrated into other furniture wall units in other residential areas (scaling of the system).

Aside from the experimental development of the RmR prototype, the project aimed at covering all necessary levels of value creation i.e. marketing, development, manufacturing, installation and maintenance. Besides the technical development dealing with embedding RmRs in business models, the option to transfer the installed terminals' information, communication and building technologies into household and nursing-related services providers was also emphasized. 


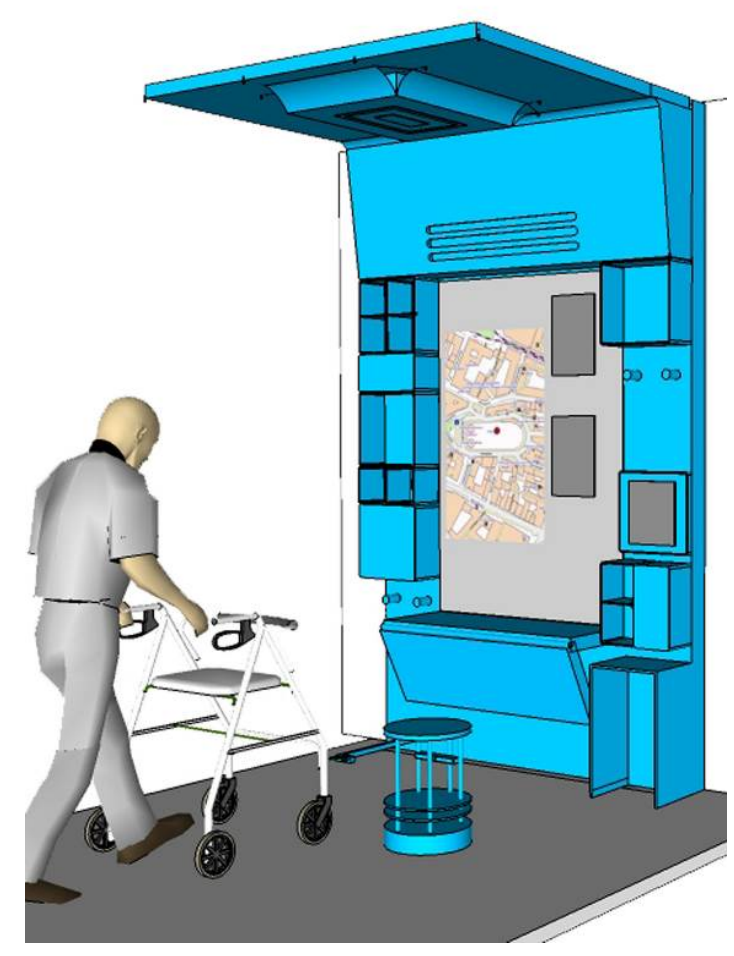

(a)

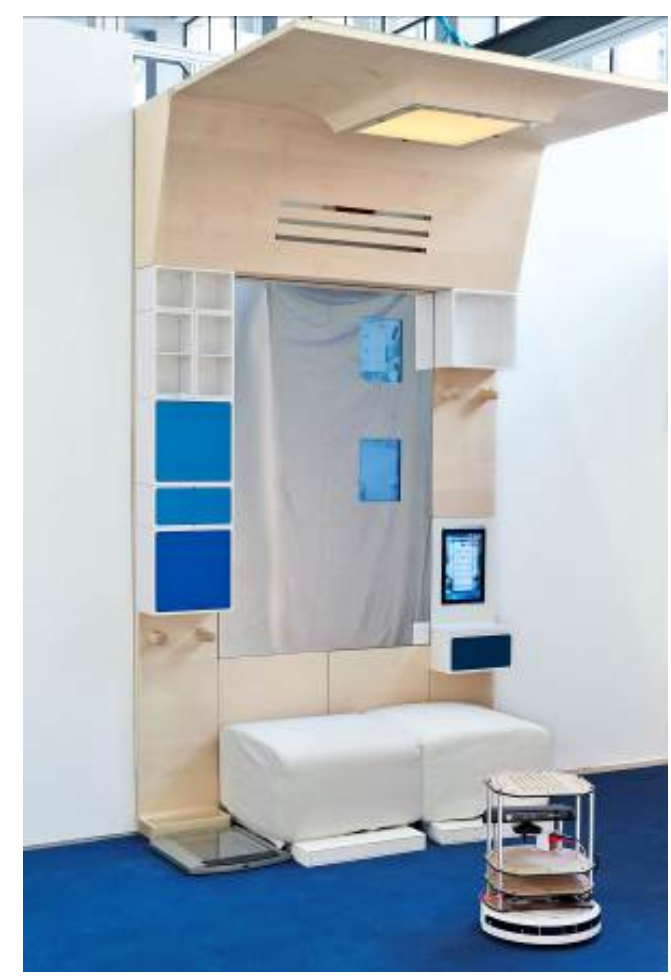

(b)

Fig.1. 1:1 System vision in development phase and prototype of the RmR

The research project LISA is located in the field of translational research. The methodological objective of the project is to translate the approach of RmRs into specific applications within the residential environment. By following an iterative experimental procedure, not only initial application possibilities were generated by the project, but methods were also tested for the systematic training between the technical bases and the concrete, industrially usable use scenarios.

To ensure scientific progress in each phase of the project, the project was divided into four phases of development. In every phase of development, analyses were first performed, followed by a concept initial development, a specification phase and a final collection of user feedback and evaluations. User feedback and evaluation formed the starting point for the follow-up project LISA "Habitec" (habitat, Bits and Technology in an Ageing Society) which deals with installing RmRs within the entire apartment, thus expanding the current system to further Life Centers.

Thus, these RmRs designed in a way that they do not replace existing walls, can facilitate cost effective affordable solutions for elderly people, even those with limited income or resources. Furthermore, the RmRs are planned in a way that for each life center in the home environment, an individual terminal is deployed. Thus, each terminal is designed and developed according to the associated life center and according to the technologies and services that the user requires with respect to the corresponding ADLs.

These first 6 Life-Centers were identified in an apartment and sorted according to their complexity ( 1 = not very complex, 6 = most complex): 1 Entrance, 2 Relaxing, 3 Living, 4 
Working, 5 Food and 6 Body Care. The evaluation criteria consisted of the installation complexity (piping, cabling, necessary subsystems, design effort, etc.). As far as the project LISA is concerned, the Entrance terminal was developed, as this was the least complex one, and additionally will enable the knowledge and experience gain for the further development of more complex terminals for Life Centers 2-6.

Several prototypes have been developed and evaluated in terms of ergonomics and ease of use in the above-mentioned iterative and experimental 4 phases of development: Phase 1: Interview with elderly people regarding the design and multidimensional assistance, Phase 2: Development and evaluation of the first mock-ups using an age simulation suit (Figure 2a), Phase 3: Evaluation of prototype 1 with real test users (Figure 2b), and Phase 4: Long-term Evaluation of refined prototype (prototype 2) in a real home environment (Figure 2c).

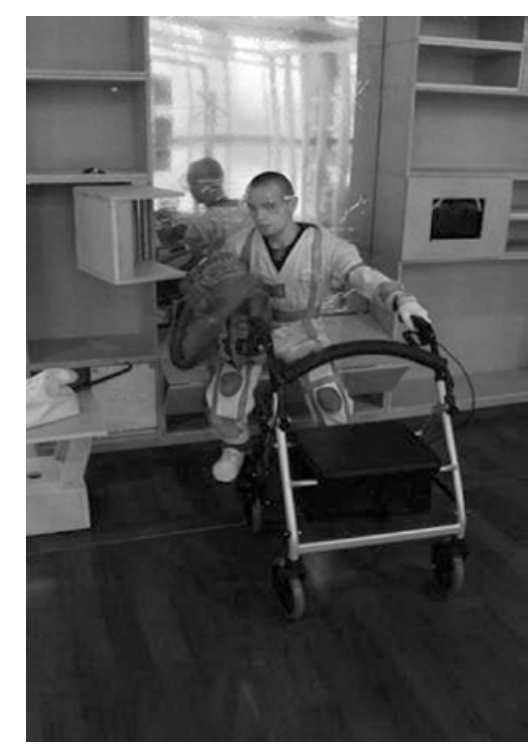

(a) Development Phase 2

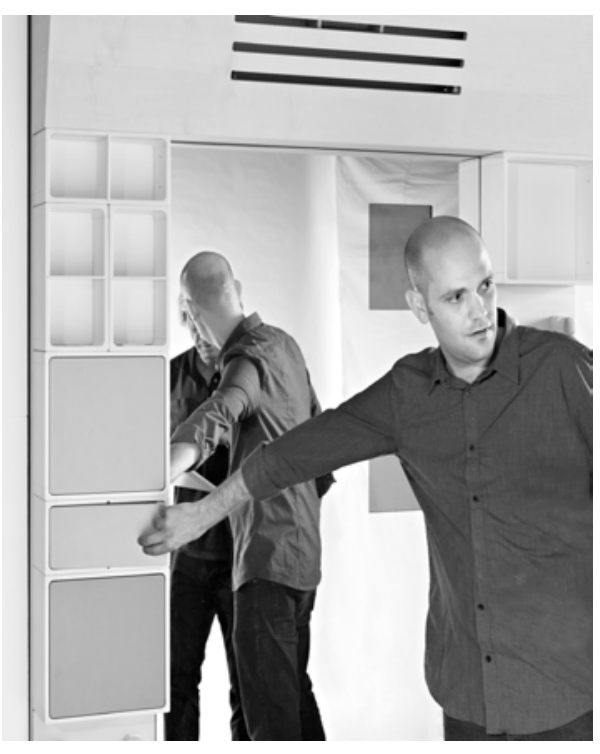

(b) Development Phase 3

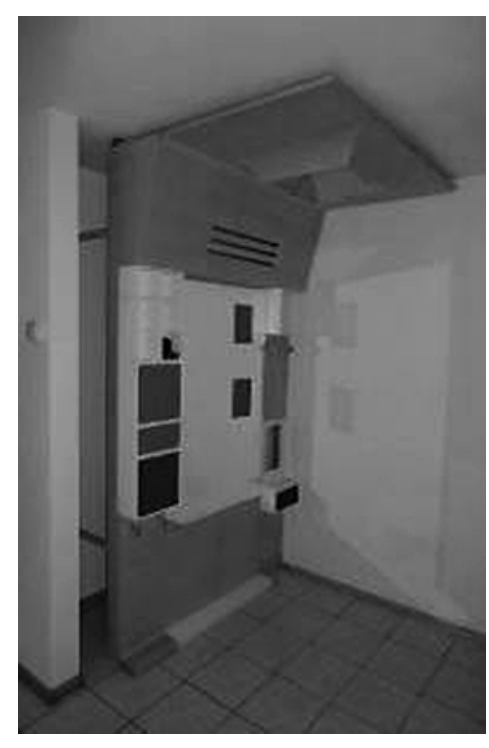

(c) Development Phase 4

Fig.2. RmR Development Phases

\section{Experimental development of the overall system}

The RmRs combine the technical fields of robotics and architecture to enable the independency of elderly people upon performing ADLs. In the first development phase of the project, a modular configurable RmR was developed in the form of a wall element for the apartment entrance and equipped with the necessary assistive functions (sensors and mechatronics), which allows the inhabitants to more easily perform the inter-related ADLs.

\subsection{The Terminal Approach}

In LISA, it was initially foreseen to discreetly embed all assistive functions in a terminal wall system. Considering residential environments and renovation processes, in terms of the ageappropriate adaptation of existing buildings, particularly in Europe and especially in Germany, the 
volume of construction associated with the current building stock already exceeds the volume of new construction. This shows that an approach that requires serious adaptation and refurbishment of the interior arrangement and layout, in order to transform existing walls into smart-walls (Figure 3a), is impractical because it requires expensive demolition of the existing installations.

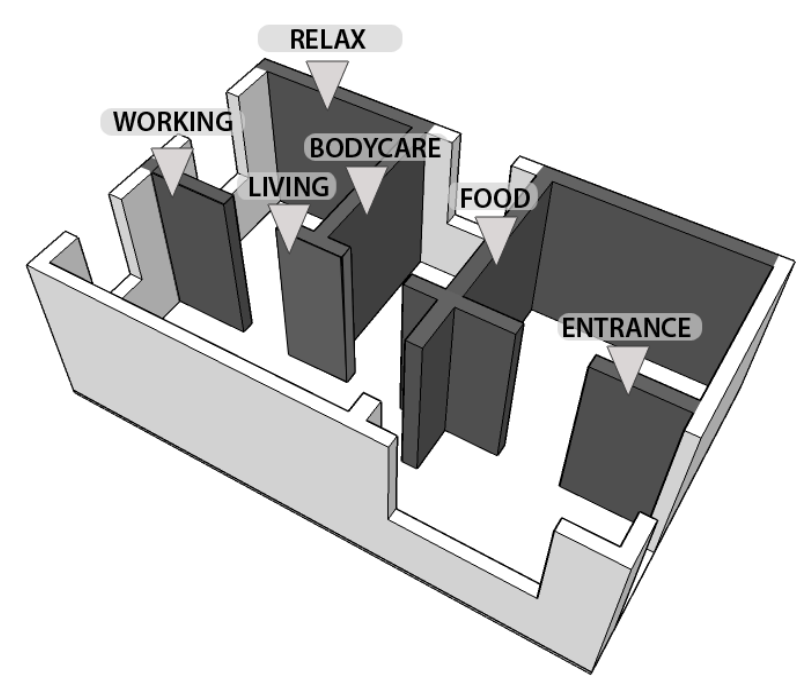

(a) Smart - wall approach

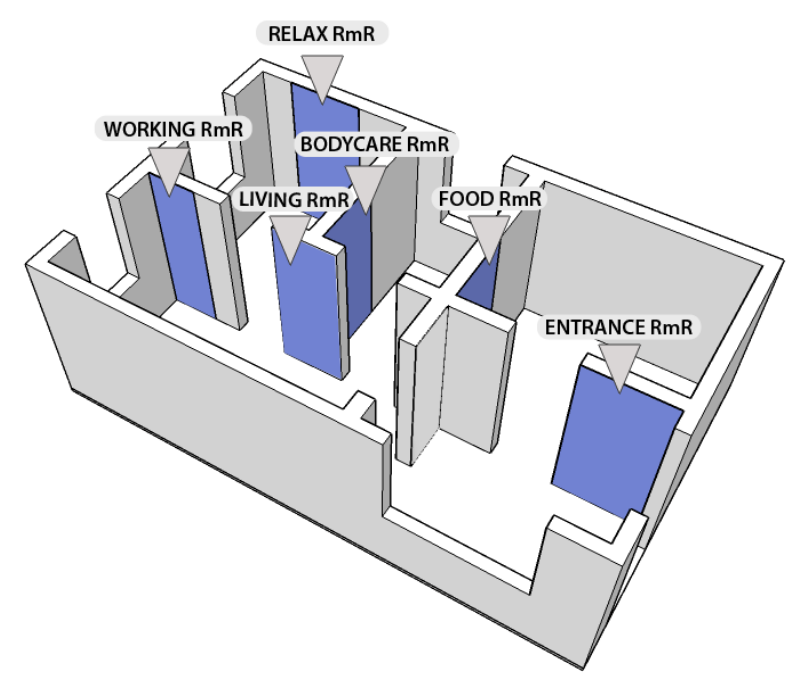

(b) Terminal - wall approach

Fig.3. Terminal-wall approach versus smart-wall

The so-called “Terminal-Wall” approach was gradually developed (Figure 3b). Based on this approach, the LISA project focused on pre-embedding assistive functions in a modular element as a kind of furniture or cabinet, which can be later installed in the room without requiring refurbishment of the existing interior. Because of the concentration of the assistive functions in a single terminal, a compact element arises which is efficiently produced and can be easily installed within the apartment. As the "entrance-terminal" explicitly focuses on specific services in the residential environment, we refer in this article to RmRs, which are in contradiction with complete robotic environments. With this approach, the general problem of user acceptance on such a system, that is typical of such approaches, is bypassed. The terminal is placed in a small area in the flat in relation to the structured environment, which forms the basis for the function and interaction of assistive subsystems.

\subsection{The Cockpit System and Partial Structuring of the Environment}

Similar to an ergonomically optimized cockpit, physical and information technology functions are arranged in a three-dimensional architectural space so that a smooth and optimum operation of processes and activities is possible: a partial highly structured room is realized within the home environment that enables the automation of assistive functions and the efficient 
integration of robotic subsystems (Figure 4). Based on the design of a Ceiling Panel, a Wall Panel and a Floor Panel (which represents a highly structured installation space corresponding to the projection of Robot Oriented Design [29]) a three-dimensional space is introduced. In this space:

i. processes and activities widely known and partially standardized are possible

ii. the geometric and functional configuration of the environment is known (all functions and shelves can be plugged to the RmR control system and automatically register when installed)

iii. integrated sensor systems (for example integrated RFID readers) are able to recognize and locate objects, persons, vital signs, etc. in a robust way.

This creates, similar to a factory environment for industrial robots, a comparatively structured space in the living environment, which allows individual robotic subsystems to assist in efficiently implementing ADLs.
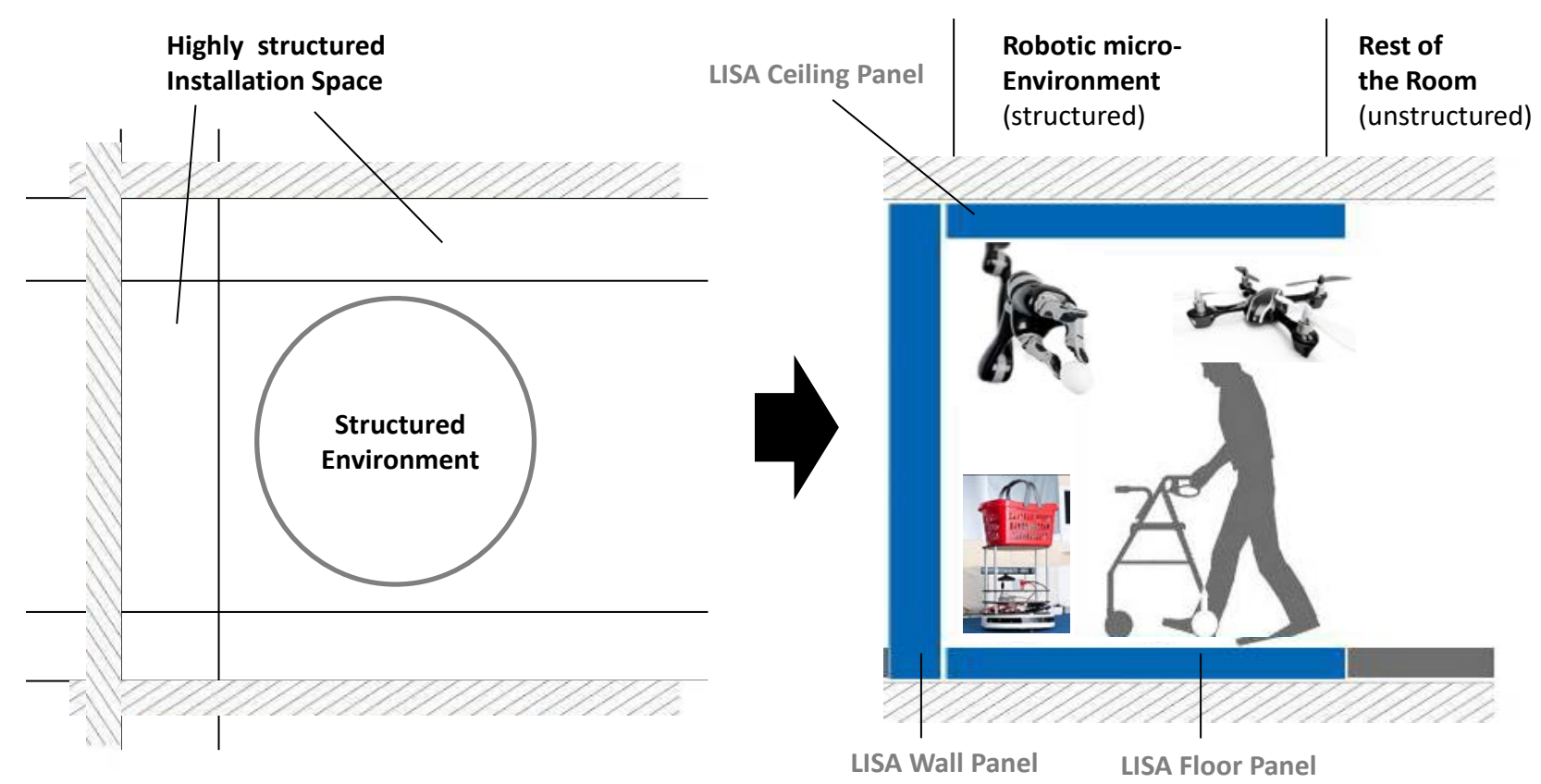

Fig.4. Basic elements of the cockpit system

The embedded assistive functions and robotic subsystems of the developed cockpit-style terminal depend on the concerned Life Center or on user-specific needs, thus reducing the complexity of the resulting system. A terminal can consist of one of these three elements or any combination. By analyzing the occurring activities and processes in relation to the ADLs in a LifeCenter, the terminal can be designed by either a ceiling, or wall, or floor module, or combinations of these.

\subsection{Multidimensional Assistance}

As a prerequisite for the development of multidimensional assistance systems, the limitations for elderly people can be subdivided in the following basic categories of the LISA project: 1) sensory limitations, 2) motoric disabilities, 3) social constraints, 4) cognitive impairments, 5) 
mental impairments and 6) somatic limitations. The simultaneous action of various limitation areas is referred to as multimorbidity and is frequently encountered in the ageing process. To counteract multimorbidity and to ensure that ageing persons can still spend an independent and self-determined life in their familiar environment without affecting their social activities, living environments must be designed in a way that they support a flexible integration and a simultaneous interaction of different assistance systems in combination with architectural and structural measures and physically and informational functional and active properties of the living environment. Therefore, the RmRs developed in LISA concentrate a variety of support functions for multidimensional assistance for each living area (Life-Center/Terminal Approach).

\subsection{Systematic Transfer of ADLs into Assistance Functions}

To get an in-depth perspective on the living situation and to determine to what extent the limitations actually affect the living situation and independence, in the initial phase of the project, the focus was on the analysis of ADLs and more importantly on the ability to perform those activities independently despite certain limitation combinations. ADLs, as well as the process parameters can also be captured well geriatrically [30, 31], and thus can be converted systematically into concrete assistive functions. Multimorbidity may enhance individual disease patterns and generate new problems. Assistive systems, embedded in the terminals, are able to counteract multimorbidity and, consequently, the loss of physical and mental performance. Residential environments must support a flexible integration and interaction of different assistive systems in combination with architectural and ergonomic measures, services and the respective members and supporters of the elderly. In this project, the authors connect the approach of multidimensional assistance and the approach of organizational ergonomics (optimized arrangement of various functions in space, which allows a quick, simple and healthy version of residential activities) with the analysis of the ADLs.

As mentioned earlier in section 1, ADLs represent everyday tasks people usually need to be able to independently accomplish. ADLs though can be grouped into two main categories: a) Basic ADLs (BADLs) and b) Instrumental ADLs (IADLs). BADLs [32] consist of self-care tasks, including: personal hygiene and grooming, dressing and undressing, self feeding, functional transfers (getting into and out of bed or wheelchair, getting onto or off toilet, etc.), mobility (walking without using an assistive device such as walker, cane, crutches, or wheelchair). More complex skills are defined as IADLs. IADLs [33] are not necessary for fundamental functioning, but they let an individual life independently in a community: housework, taking prescribed medications, managing money, shopping for groceries or clothing, use of telephone or other form of communication, using technology (as applicable), transportation within the community. Medical conditions and capacities of older people can be evaluated through a so-called "geriatric 
assessment”. Statements can then be made about the extent to which elderly people are able to independently perform ADLs and what needs are possible to be supported. Through a detailed analysis of the activities in the individual Life Center, with the help of an analysis of movements of older people in the Life Center as well as appropriate surveys of elderly people upon testing the respective prototypes for each Life Center, the most important and, thus, generated assistance functions were identified gradually and systematically.

\section{Proposed system architecture}

This section describes the operation of the overall system as well as the technical details of the assistive functions. The system is constructed in a modular setting, as previously described, so that more assistive functions and robot subsystems (also provided by suppliers) can be easily integrated in the future. The embedded functions comprise (Figure 5):

- an intelligent ambient lighting system

- a mobile robotic rover (Turtlebot) as an intutive user-machine interface

- an air quality purifying system

- a standard touchpad interface (tablet PC) for real-time display of navigation and weather information

- a reminder-alerting RFID-based monitoring system

- user vital signs display and measurement system

- a shoes putting on/taking off assisting system

- assistive standing-up, sitting-down system

Figure 5 depicts the currently implemented subsystems. The features range can be easily extended or modified due to the modularity and customization approach that was followed during the prototype's design phase, which enables a "plug-n-play" installation. In Table 1, the overall features of the proposed system are listed, according to the system and subsystem level. The control of the RmR can be done directly on the mirror in the middle of the terminal, (via two dedicated touch screen display areas, Figure 1b) or via a tablet PC (Figure 5d). The functions mentioned in Table 1 are controlled by one of the two PCs (LISA 2 PC, Figure 6). The second PC (LISA 1 PC) offers the possibility to retrieve weather information or to access the World Wide Web (WWW). Via the tablet PC, the user can retrieve and display information such as weather data or recorded vital signs measurements. Additionally, via the tablet PC, the user can also access and control all functions of the RmR, which can conveniently be carried out within the apartment. The system architecture is presented in detail in Figure 6. 


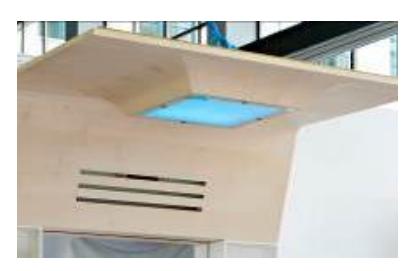

(a) Light

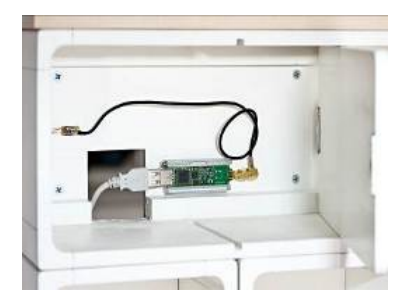

(e) RFID-based alerting system

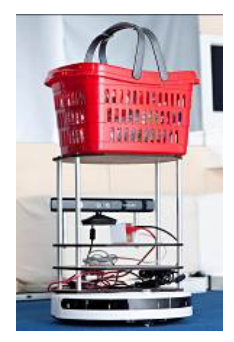

(b) TurtleBot

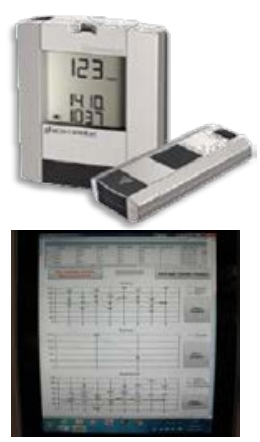

(f) Vital data recording and display

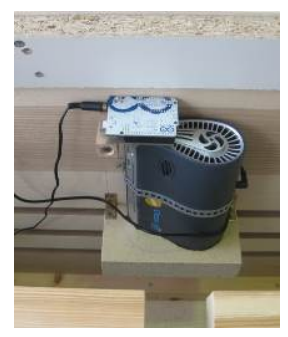

(c) Air quality purifier

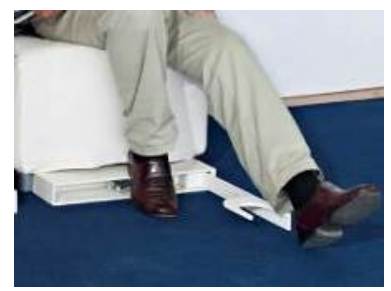

(g) Putting shoes on/taking shoes off assistance

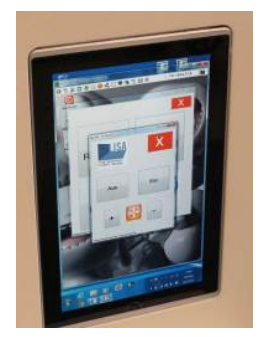

(d) Navigation and weather data

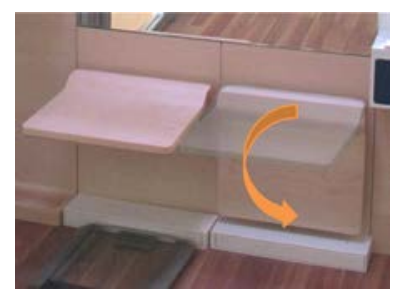

(h) Standing-up, sittingdown assistance

Fig.5. Embedded subsystems of the RmR space

\subsection{Intelligent Ambient Lighting System}

The integrated ambient lighting grid enables the ability for bio-feedback and risk-related warnings. In case of a recorded high blood pressure reading, a dark red light pattern is emitted to warn the user of possible risk situations. Similar light patterns are emitted in the event the user leaves the house while forgetting their glasses or house keys stored within the RmR RFID-enabled cabinets. 


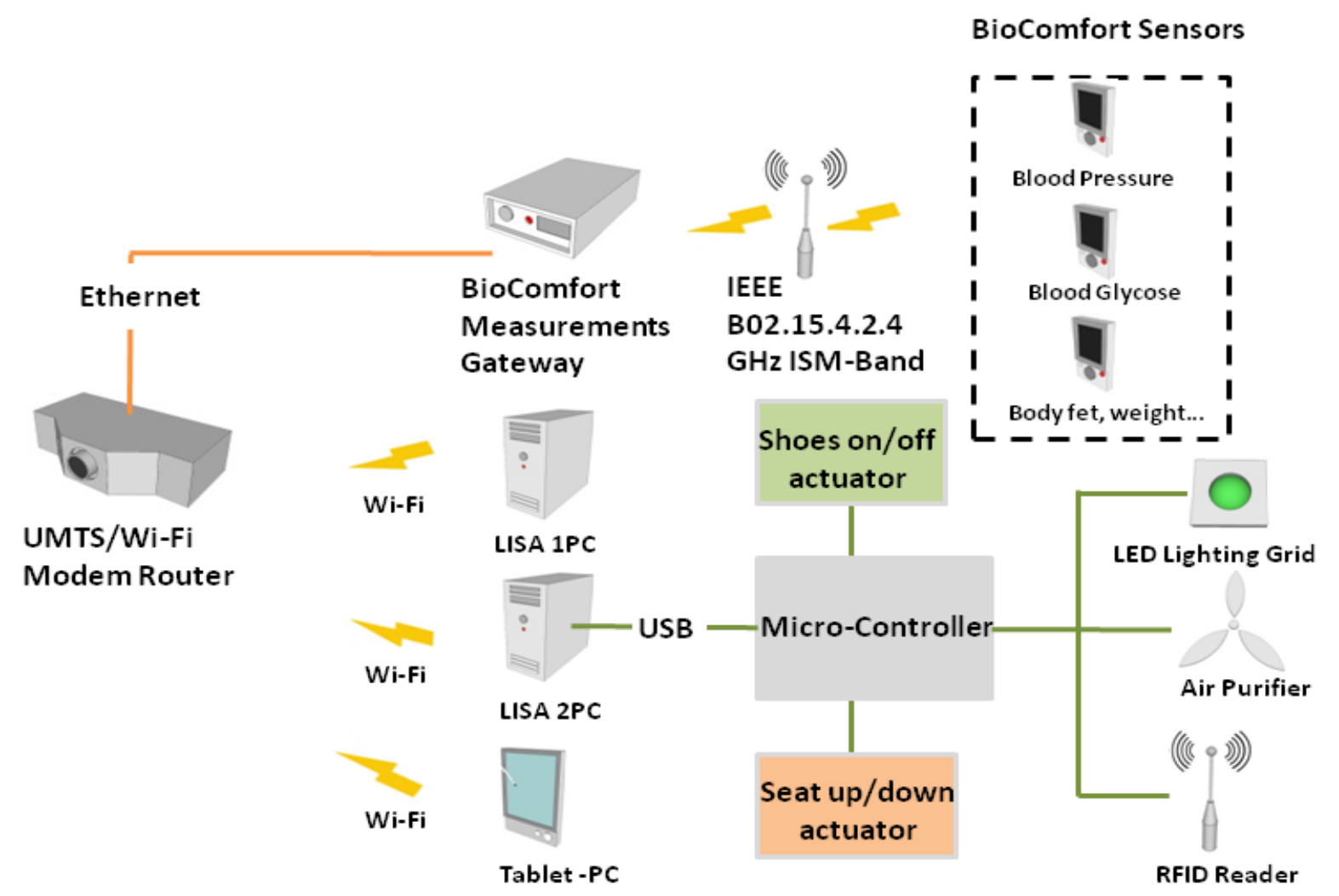

Fig.6. System architecture of the RmR

\subsection{Mobile Rover user-machine interface system}

A mobile rover, Turtlebot [34], was used to act as the medium between the user and the RmR via intuitive gestures and voice commands. Additionally, the Turtlebot, due to its autonomous navigation features, offers the ability to navigate within the user home environment simply via location-related vocal commands, i.e. “entrance”, “kitchen”, “bedroom”, etc. Elderly people can therefore be assisted upon entering the home environment in case they carry shopping bags or baskets, by vocally commanding the Turtlebot to transport them to i.e. the kitchen.

The user can communicate the required vocal commands to control the RmR functions to the TurtleBot via a standard training procedure. For this, the necessary vocabulary is recorded via an integrated microphone onboard the mobile rover. The training procedure can be repeated to extend or customize the functionality. The environment arrangement in which the TurtleBot traverses must be known to allow for autonomous navigation. For this purpose, the widespread technique “Simultaneous Localization and Mapping” (SLAM) [35] is used. SLAM enables an autonomous rover to create a detailed map of an unfamiliar territory. The TurtleBot is able to acquire visual information using its onboard Microsoft Kinect Sensor [36]. Once the map is recorded, the Turtlebot can then efficiently navigate through the home environment in an autonomous operation. Figure 7 depicts the environment 3-D reconstruction of the RmR arrangement. As a domestic environment can be considered dynamic, e.g. objects are put down for a short time, the inhabitants switch their position and furniture layouts are changed, the TurtleBot is able to update in real-time 
these dynamic environment changes, and update its map data with the current recorded arrangement to avoid obstacles that might be encountered in its path. With such a system, problems such as 3D reconstruction, current position, autonomous navigation, obstacle avoidance, etc. are efficiently addressed [37]. Further technical details and scenarios for integrating the TurtleBot as an innovative user interface are analyzed in detail and explained in [34].

Table 1: Modular functions of the RmR

\begin{tabular}{|c|c|c|}
\hline Level & System & Sub-system \\
\hline \multirow{6}{*}{ Environment } & \multirow{3}{*}{ Ambient Light } & Color temperature \\
\hline & & Brightness Variation \\
\hline & & Blinking alerting-pattern \\
\hline & \multirow{3}{*}{ Air Purifier } & Fan Speed regulation \\
\hline & & Humidity \\
\hline & & Fragrance \\
\hline \multirow{7}{*}{ Function } & \multirow{3}{*}{ Reminder } & $\begin{array}{l}\text { Alert on risk situations, i.e. leaving flat while keys are } \\
\text { stored within the RmR }\end{array}$ \\
\hline & & Irregular vital signs \\
\hline & & Main door unlocked, high ambient humidity, etc. \\
\hline & \multirow{4}{*}{ Vital Data Measurement } & Blood pressure \\
\hline & & Blood glycose \\
\hline & & Body weight/fat \\
\hline & & Heart pulse rate \\
\hline \multirow{8}{*}{ Service } & \multirow{5}{*}{ TurtleBot mobile rover } & Remote control by hand gestures \\
\hline & & Remote control by vocal commands \\
\hline & & Carrying shopping bags \\
\hline & & Emergency call \\
\hline & & Ambience Intelligence \\
\hline & \multirow{3}{*}{ Architecture Design } & Linearly actuated robotic seat \\
\hline & & Shoes putting on/taking off assistance \\
\hline & & Touch screen tactile input display \\
\hline
\end{tabular}



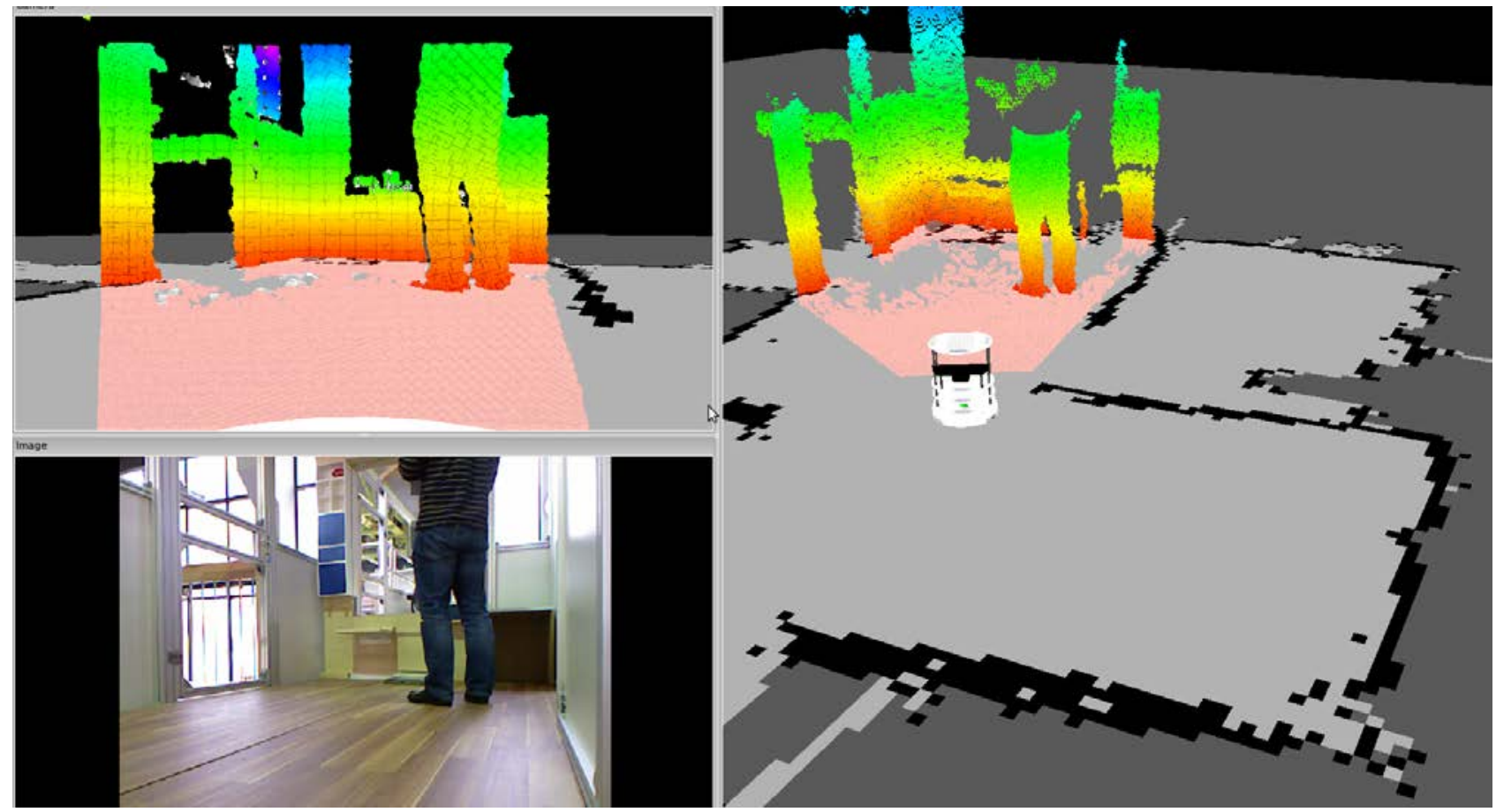

Fig.7. Turtlebot home environment 3-D reconstruction

\subsection{Air purifier system}

An air purifier system was also embedded at the top part of the RmR module. A humidity sensor and a temperature sensor attached to the same module enable the modification of the air quality in the vicinity of the terminal. The air purifier can be controlled either automatically; i.e. in the event of high humidity, increased temperature, or a combination of both situations are detected by the corresponding sensors, it switches its fan on; or by the user via the installed touch screen displays of the RmR, which act as a control panel for controlling the embedded subsystems functionality via specifically designed graphical user interfaces (GUIs). The user is able to manually regulate the air purifier fan speed, and also to completely switch off its operation if needed.

\subsection{Tablet PC mobile user interface}

Even though the RmR can be controlled via the dedicated touch screen displays on the front side of the terminal, an additional tablet PC was included in the overall system architecture to enable remote access to the proposed system through a mobile device. The user would have been limited in terms of functionality and straightforward operation if the control of the terminal would have been performed explicitly via the touch screens onboard the RmR. According to conducted field tests, elderly people expressed an interest in also being able to control the embedded functions of the RmR remotely, via the use of a standard tablet PC device. By including a mobile device in the control interfaces loop, this extends the potential of the embedded services and enables an efficient remote operation of the terminal. 


\subsection{RFID-based monitoring and alerting system}

The RFID (radio-frequency identification) allows identification and localization of items that are tagged with an RFID transponder. The RFID technology is much more like a barcode, but requires less space because there are no actual bar codes used. Generally, RFID tags can be divided into two classes: active and passive. In the RmR approach passive tags were used, which means their necessary power supply is excited by the RFID antenna signal strength. Since objects can be identified and their position concerning individual cabinet elements can be assigned, a structured "work environment" is enabled. RFID technology [38, 39] was used to record and monitor users' personal items and belongings, enabling a novel localization scheme by the generated item inventory. Using passive RFID tags attached to the users' personal items, the RmR can perform a cross-checking upon the user entering or exiting the apartment, and specifically warn for irregularities. The RFID antenna was embedded into specially designed cabinets of the RmR terminal, thus allowing the generation of an inventory of the items the user stores within them (Figure 8).

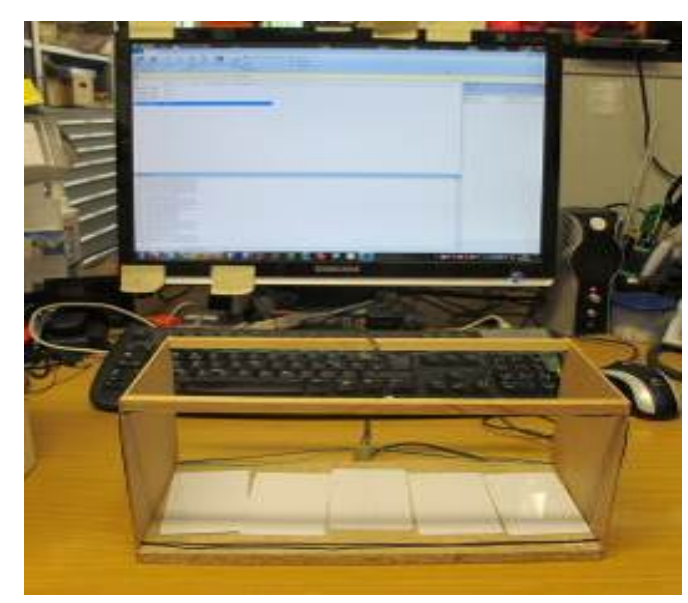

(a) Programming of RFID tags

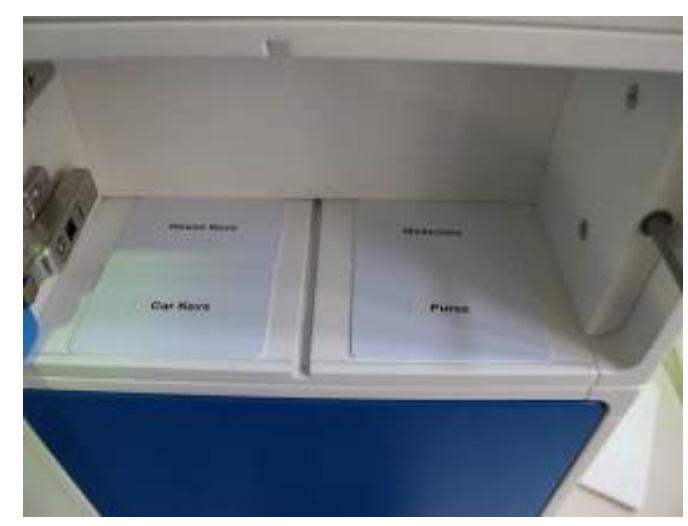

(c) RFID Tags

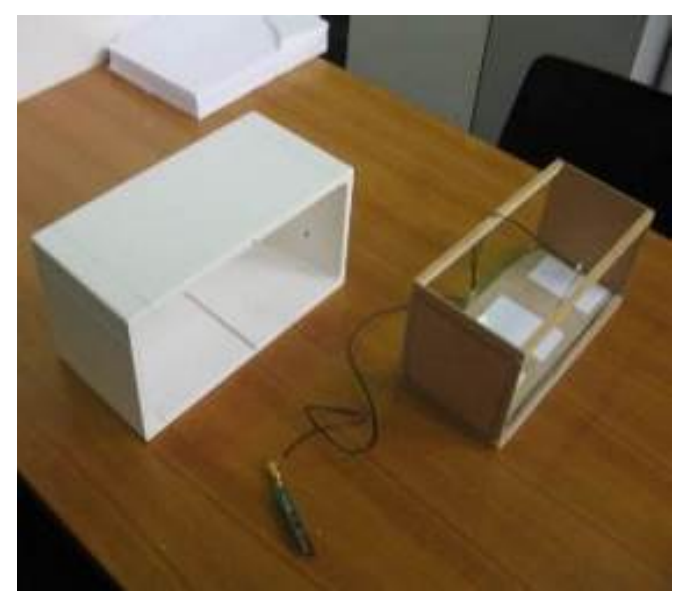

(b) Functional test of the RFID Tag

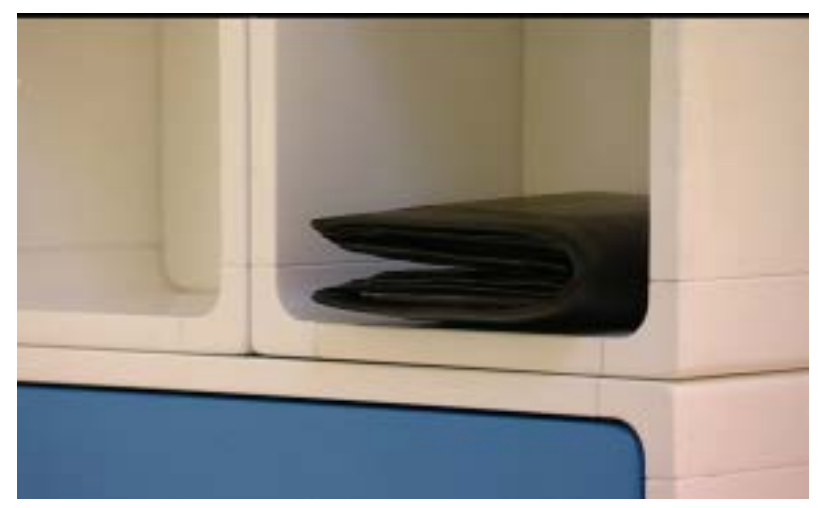

(d) Wallet with RFID Tag

Fig. 8. RFID antenna invisibly integrated into individual cabinet elements 


\subsection{User Vital sign monitoring and alerting system}

A significant feature of the proposed system is the tele-monitoring of users' vital signs and health statuses. The monitoring is performed using a diagnostic scale, which provides a bioelectric impedance analysis (B.I.A.), measuring body fat, body water, and muscle mass, a wrist measurement device for heart rate and body temperature, as well as a blood glucose meter [40]. All three devices are wirelessly interfaced to a measurement gateway, in order to enable wireless health status data acquisition and storage. Once the user performs a measurement, the reading is instantly wirelessly transferred to the measurement gateway. The gateway has the ability to store the last 1000 measurements locally, as well as to push the measurements database to an online website through a secure transfer protocol. This allows the user to be remotely checked by a physician accessing the vital signs measurements online database, simply by using a set of predefined credentials (i.e. a username and a password) [41]. The measurement of these vital signs is of particular importance for the elderly as the leading causes of death in Germany are coronary heart diseases (CHD), heart attacks and cardiac insufficiency [42]. These diseases often result from the poor lifestyle of the persons concerned. Due to lack of exercise and improper nutrition, some people develop type 2 diabetes. As this disease often occurs in old age, it is therefore called "adult-onset diabetes". Diabetes promotes the development of KHKs [43] because the high concentration of sugar in the blood damages the blood vessel walls, thereby forming slight vascular deposits that can eventually lead to vascular occlusions. A high age, smoking, obesity and high blood pressure also increase the risk of heart attack [43]. Obesity, type 2 diabetes and high blood pressure are "creeping" diseases, i.e. initially asymptomatic, which often have the consequence that just after first damages occur (e.g. angina pectoris) a treatment takes place. To reduce the risk of death by KHKs, it is important to ensure full compliance with diet or medication.

\subsection{User functional assistance by robotic actuators}

The user can be assisted upon putting on or taking off shoes with less effort, via an embedded mechanism on the bottom of the RmR terminal, which is deployed using a rotary actuator. The user can place their foot into the respective mechanism opening, which secures the shoe firmly, and by pulling the foot upwards, the shoe is removed. The same can be performed upon putting the shoe on. Finally, a linearly actuated robotic seat assists the elderly upon sitting down and standing up, by altering its current height according to the user requirements was implemented in the proposed RmR to allow also the easier implementation of functional transfers, i.e. from wheelchair/rollator to the RmR seat (Figure 5). 


\section{Evaluation and User Feedback in the Different Phases of Development}

To ensure scientific progress in each phase of the project and to not focus the user studies only at the beginning or at the end of the project, the project was divided into four phases of development. Overall, the methodology in the solicitation of the user feedback or an evaluation of a more explorative way in the early phases of development enables the successful feedback and evaluation of the outcomes in the later phases. User feedback at all phases of development was found to be necessary and determining, since by following such an approach the end product gets finally developed based on the accumulated and evaluated undoubtedly important users inputs. When a user evaluation is considered for the first time during the final development phases, the overall usability and potential of the product cannot be fully exploited, or the specifications cannot be greatly altered. Instead of compromising a products usability and acceptability by end users, user feedback must be considered from the early phases of development, to enable a more appropriate and efficient design, implementation and realization strategy.

Since the project aims at future novel intelligent system solutions, by installing RmRs into a home environment to have a dedicated consistently explorative character, the same exploratory character has also been emphasized in the last phase of development. An explicit adjustment to market needs is planned for the forthcoming project, LISA Habitec, as the basis for the methodical transfer of the approach has already been provided in concrete applications in terms of in the current LISA translational research. Additional resources can be used to expand the current RmR system to the other remaining Life Centers of the home environment.

\subsection{Development Phase 1}

The demand analyses with the industry project partners formed the starting point for the project, enabling the need for a flexible terminal system for homes, apartments, retirement residences and hotels with features and customization options, particularly focused on the ageing society target group. Preliminary studies were conducted for the development of compact space elements or room-in-room systems that focus on assistive functions for the elderly. First, the activities were identified in each living area via a) analysis of regular apartments configurations, b) experimentation with an age simulation suit, and c) surveys of potential users. As it was observed that the classic conceptions of living have fundamentally changed, the consortium proceeded to no longer referring to rooms (kitchen, bedroom, etc.) but to a sub-division of these, Life Centers. Life Centers are strategic locations within an apartment, which do not have to be separated by walls (barrier-free) and are constantly changing over time, in contrast to the classical understanding of space need continuum. By means of a cost-benefit analysis, different approaches were evaluated to 
establish these Life Centers with different functions (from classic wall systems to furniture systems and up to cubicles in space systems) with respect to the project objectives. Finally, the previously described (section 3) terminal approach was derived from this assessment.

The terminal approach was then evaluated in workshops and through interviews with the targeted user group and discussed in an exploratory way, concerning the potential and desired functions of each Life Center. For further refinement of the Life Center, the following categories of parameters were identified (table 2) as highly relevant for the maintenance of daily activities. The individual parameters were systematically translated into functions in development phases 2 and 3.

Table 2: Identified Parameter Categories

\begin{tabular}{|c|c|c|}
\hline No. & Category & Parameters \\
\hline 1 & $\begin{array}{l}\text { User remains in every situation in the foreground of } \\
\text { the situation }\end{array}$ & $\begin{array}{l}\text { Clarity, Orientation, Simplicity, Individual arrangement of } \\
\text { assistance functions }\end{array}$ \\
\hline \multirow[t]{5}{*}{2} & Well-being & Recreation \\
\hline & & Consideration of the individual "lifestyle" \\
\hline & & Naturalness \\
\hline & & Homeliness \\
\hline & & Comfort \\
\hline \multirow[t]{3}{*}{3} & Functionality & Ergonomics (anthropometric/biomechanical) \\
\hline & & Organizational ergonomics \\
\hline & & Cognitive ergonomics \\
\hline \multirow[t]{3}{*}{4} & Security & Safety \\
\hline & & Security \\
\hline & & Health \\
\hline \multirow[t]{4}{*}{5} & Multimodality & Light \\
\hline & & Audio \\
\hline & & Surfaces \\
\hline & & Odors \\
\hline
\end{tabular}

\subsection{Development Phase 2}

Instead of developing all Life Center terminals as partially functioning primitive mock-ups, it was decided to fully develop a single Life Center functioning terminal in detail. Thus, a physical and informational platform as well as requirements and approaches could be developed that can be later transferred to the remaining Life Centers. The terminal for the entrance Life Center was selected for development. In comparison to other Life Centers, an enormous variety of possible activities and assistance functionalities could be identified with less complexity. The necessary user 
needs were translated into a set of requirements, and finally, into design parameters. The modularity of the system was optimized using a design structure matrix approach. To ensure an adaptation of needs of the terminal according to the budget and the users' needs, and further, to ensure the future expansion of this system beyond the project scope, particular focus was given to the modularity.

Finally, two possible configurations were built as 1:1 scale mock-ups, which were then tested on the basis of ergonomics and usability with an ageing simulation suit. In this case, user-leaving ("Departure Management") and user-arriving ("Approach Management") were identified as the two main scenarios dealing with the entrance Life Center in the residential environment. These were then broken down into sub-activities that are typical for the entrance area. Each of these activities was assessed by means of the ageing simulation suit with four different cases of user disability (1Healthy, 2-No walking aid, 3-Walking aid needed, 4-Wheelchair needed). Figure 9 shows examples of the “Approach Management” evaluation results for the two mock-ups during development phase 2. The results were used to identify the limitations and difficulties elderly people with different degrees of disability face upon performing activities of daily living in the entrance area of the apartment [44].

During the evaluation, it was noted that if a mobile rover can be introduced to assist upon the user entering or exiting the apartment, i.e. to carry the shopping bags or other heavy items, the elderly can perform other tasks involved in ADLs more efficiently. Furthermore, the experiments showed that assistance in sitting down or standing up, assistance putting on or taking off shoes, an intelligent reminder system, as well as an appropriate lighting source, can effectively contribute towards an ambient support system solution. Analyses also showed that the "Departure Management” system should include the ability to cross-check against user vital data records, and also provide appropriate mobility goals, i.e. maps and navigation instructions.

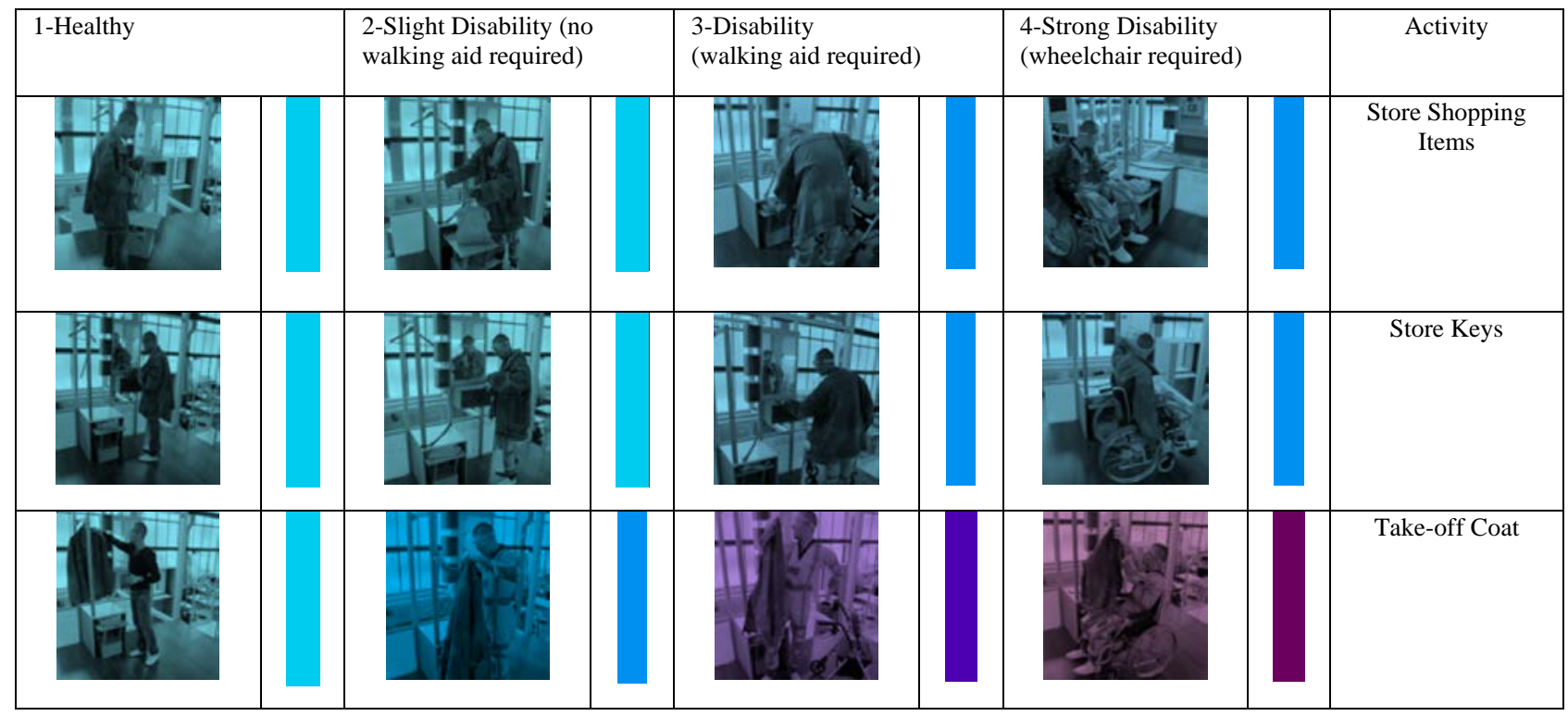




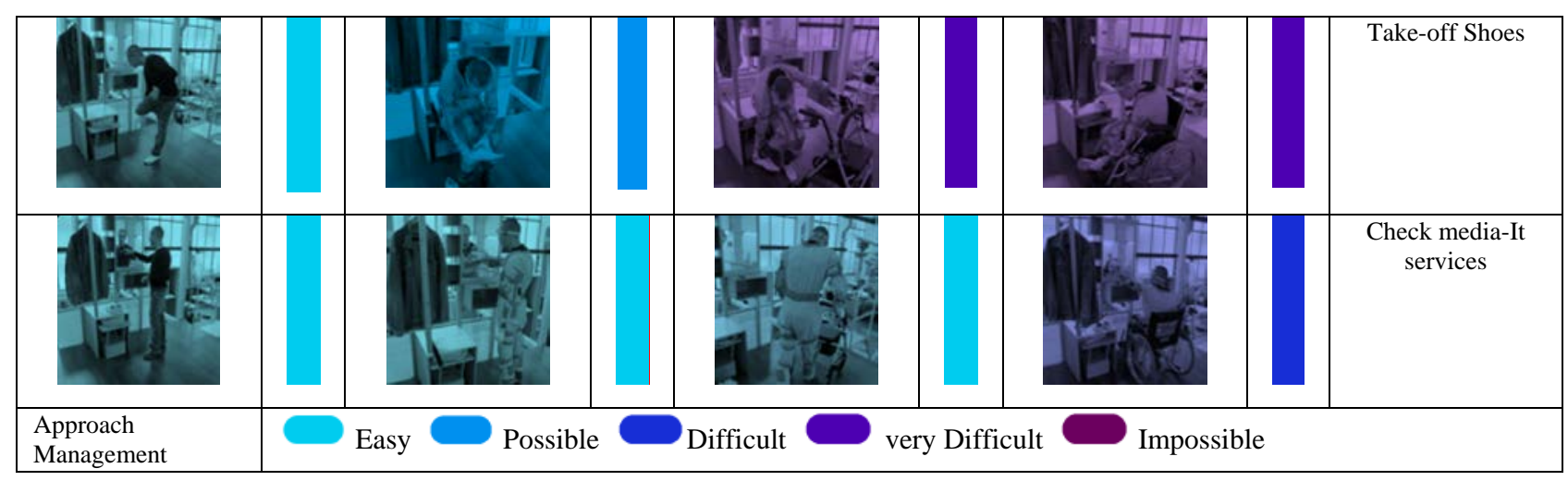

Fig.9. Approach Management Experiments Evaluation

\subsection{Development Phase 3}

Within this phase, the concept development in phase 2 was improved (modularity as well as in terms of user adoption). Subsequently, the system was further detailed and finally fabricated with all its functions as a 1:1 scale terminal. A user testing phase was performed in a controlled laboratory environment in order to optimize system functionality (Figure 10). Six test subjects participated in the study ( $4 \mathrm{x}$ male, $2 \mathrm{x}$ female) with an average age of 66 years. Two of the test subjects lived by themselves in an apartment or house, and four lived together with their partner. All 6 test subjects were still largely independent in accordance with the specifications. After the presentation and use of the system according to a predefined sequence, the test subjects were asked to complete a structured feedback questionnaire form, including relative issues and various rating scales. Furthermore, comments and suggestions were solicited and discussed in an open-discussion during the use. The comments received were diverse, but focused on the fact that the system is still far too driven by technology, and that the benefits and practical applications have further potential. All functions were rated more or less useful. In particular, the RFID-function as a reminder and connection to medical services was positively reviewed. It must be emphasized that the RmR comprises an acceptable assistive technology system. Typically these technological solutions are declined in older ages, but its medical related functions are important and easy to use.

Regarding the tablet PC control interface of the terminal, although the specially (age-oriented design) developed GUIs were rated as understandable, the evaluation showed that with increasing age, innovative and intuitive gestures or speech-driven interfaces are encouraged. 

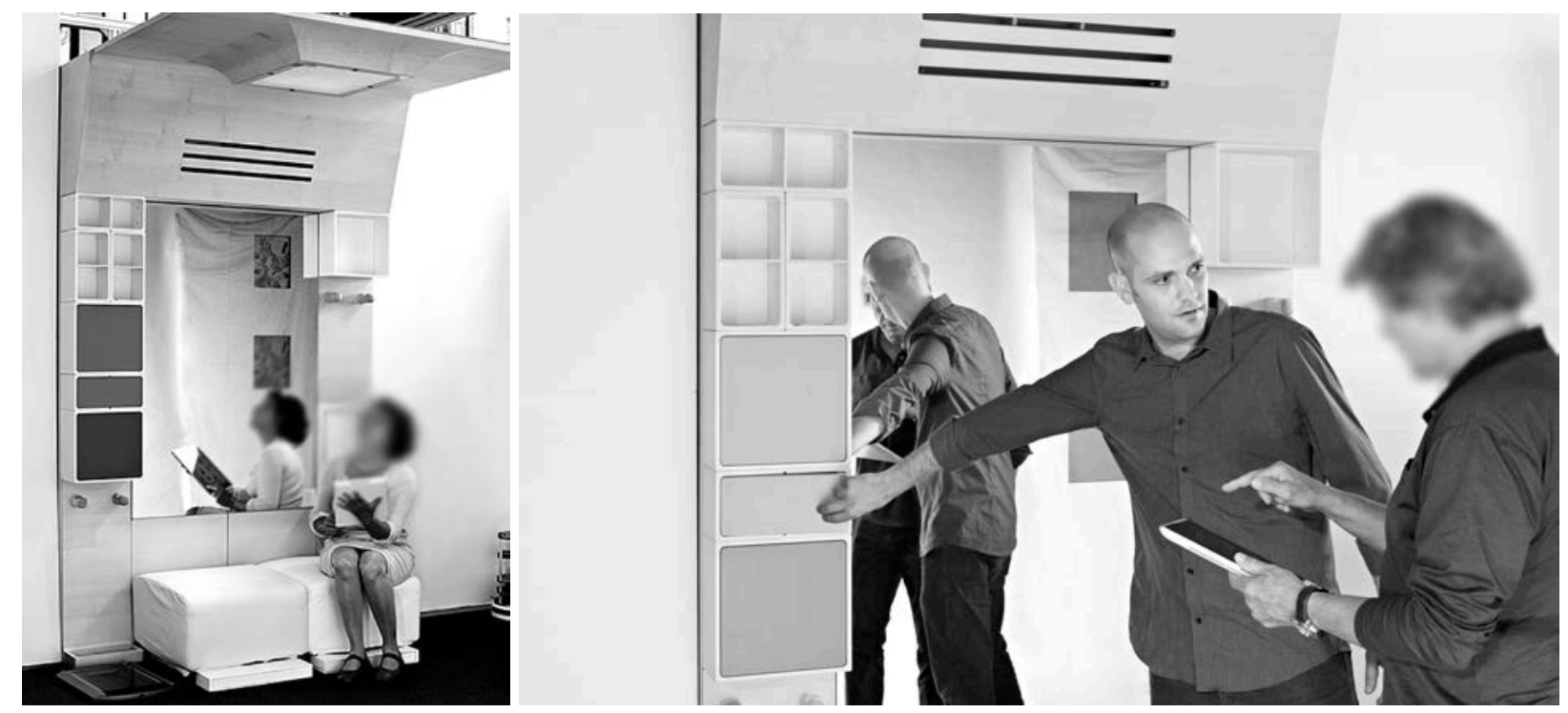

Fig.10. Tests with real users in the controlled laboratory environment during Development Phase 3

\subsection{Development Phase 4}

In this phase, first individual functions were improved based on user feedback obtained during the earlier development phases. In particular, the GUIs were refined to offer enhanced cognitive support (i.e. intense colors, different shapes, etc.) and novel control methods including gesture-driven and speech-driven operation to enable an intuitive human-machine interface. Additionally, to enhance the ease of installation and rapid deployment of the RmR, installation tests were performed (Figure 11). More specifically, the pre-assembled RmR terminal was uninstalled (Figure 11a), transferred (Figure 11b), and successfully re-installed (Figure 11c) within the same day from Germany (Munich) to Italy (Bolzano). After considering the feedback received from the 2-person installation crew, the consortium strongly believes that in the future, in extreme cases, an entire apartment can be equipped within 4 hours of installation time with RmR terminals for all 6 Life Centers and thus can be straightforwardly "converted" into an assistive environment. The installation requires a simple fixture of the metal frame to the wall, a power supply, and a live Internet connection. Due to the prefabricated approach, all required services and technologies of the RmR, are pre-embedded, tested, refined and finalized during the fabrication phase. No user related on-site refinement or calibration is required. This strategy exploits the "plug-n-play" notion of the proposed system. 

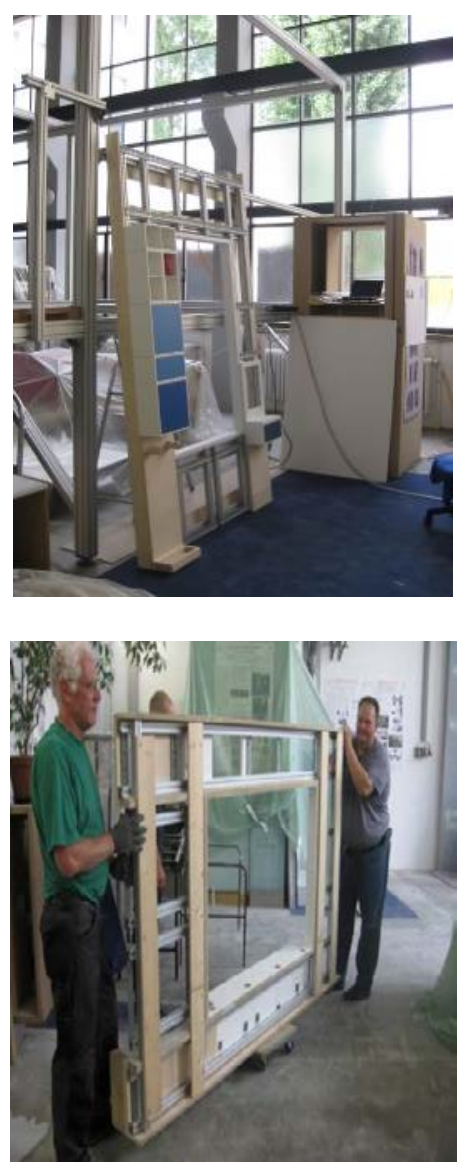

(a) Uninstallation in Germany (Munich)
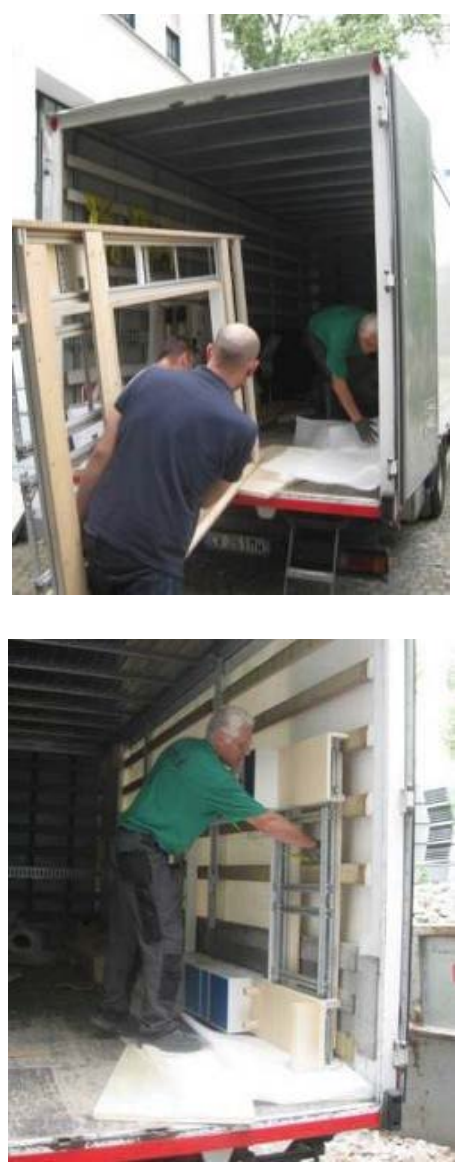

(b) Transport
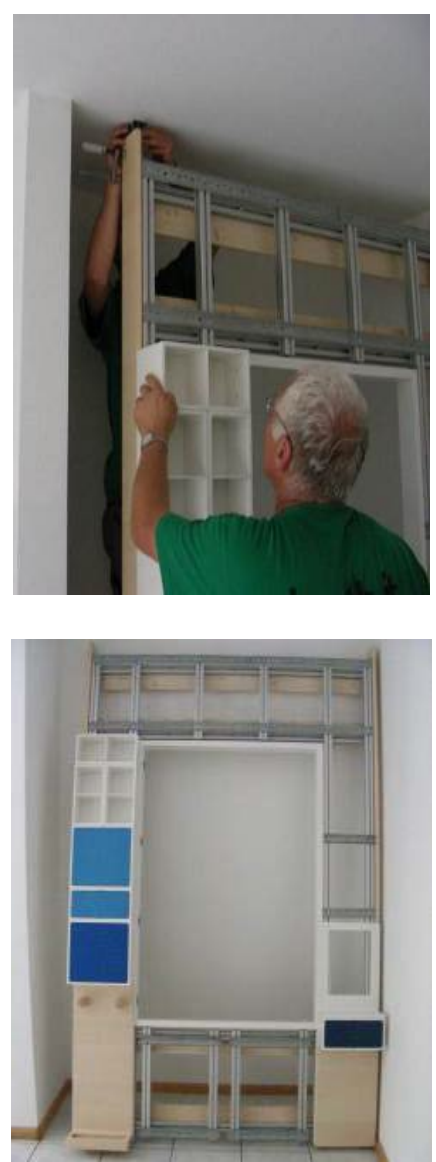

(c) Installation in an appartment in Italy (Bolzano)

Fig.11. Installation of the proposed RmR within the same day in Italy (Bolzano)

\section{Embedding of the Approach in a Product-Service System}

Besides the experimental development of the prototype and the embedded assistive functions, another aim of the project was to cover all value creation levels for the marketing, development, manufacturing, installation and maintenance. In addition to the "technical" development, highest priority was given to embedding the $\mathrm{RmR}$ in business models and to be able to transfer to appropriate providers within the building technology, household and care-related services.

\subsection{Design for Mass Customization}

The RmR, developed in the LISA project, represents the base of a robotic service terminal that supports elderly people through its functions, in the area of prevention and wellness. The modularity of the RmR on the environmental level, functional level and service level is completely adaptable to user needs. Due to the modular approach, the developed standard functions can be adapted or replaced later by customized functions when required by specific user circumstances when the system is brought to market. Thus, a RmR can be initially equipped explicitly with the most important functions, to allow for a low purchase cost, and can later be retrofitted with additional assistive functions. 
Figure 12 outlines the modularity of the system and shows that the RmR is customizable on the total system level (size and configuration of the RmR), the arrangement of individual elements/cabinets level and the level of electronics and sensor infill embedded in the elements/cabinets. The electronics infill of the cabinets can be exchanged by removing the removable cover in the cabinet. The whole cabinet can be removed from/ added to the RmR base frame using connectors. Furthermore, each cabinet is connected to the base frame by a USB connector, which supplies the electronics infill with both electrical power and information.

\subsection{RmR as a Service Platform}

The integrated IT communication elements of the terminals make it possible for service providers, such as providers of ambulatory nursing, to provide their care and emergency services, and thus be able to offer an intensive and customer-oriented service "on-demand" without a permanent physical presence. The LISA assistive terminals not only provide value-added elements, but also integrate information and communication technology as a platform for a wide range of local services. For example, it is planned that the modules offer the opportunity to local home care providers or ICT health services, to improve their services using the proposed RmR terminals as a test bed. The development of information technology in the health sector, and in particular towards eHealth, must be considered (tele-monitoring, tele-care, tele-therapy [45]). While the proposed LISA terminal does not aim, in the first phase, to medical services, but on services from the nursing field and household sector, the system can be considered an intermediate step towards the integration of tele-medicine and tele-consultation services in the residential environment. Demographic change and the increasing proportion of elderly people will influence the development of digitally assisted services of any kind, with respect to the home environment. Many elderly people who follow the trend and want to live independent, healthy and fit, manage their lives with the help of assistive gerontechnologies. Terminals equipped with micro-system technologies in the living environment enable an optimal user interface for initialization of these services.

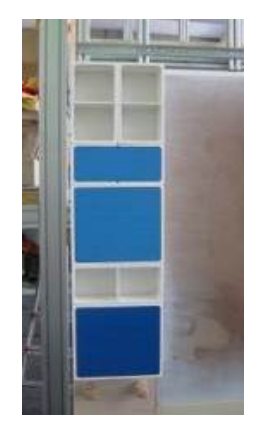

(a) Modular distribution

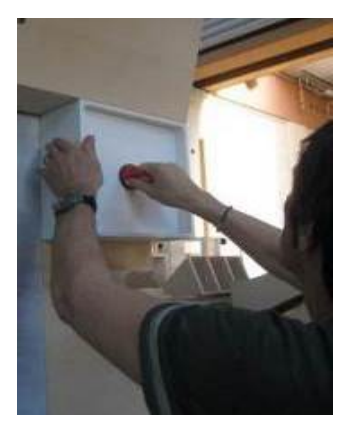

(b) Easy replacement

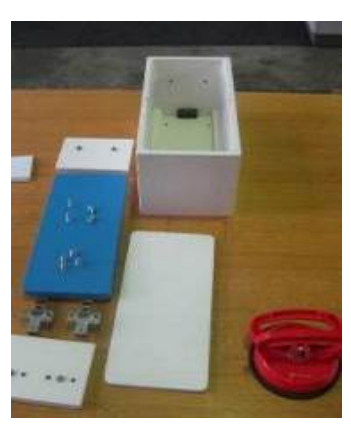

(c) Modular components 


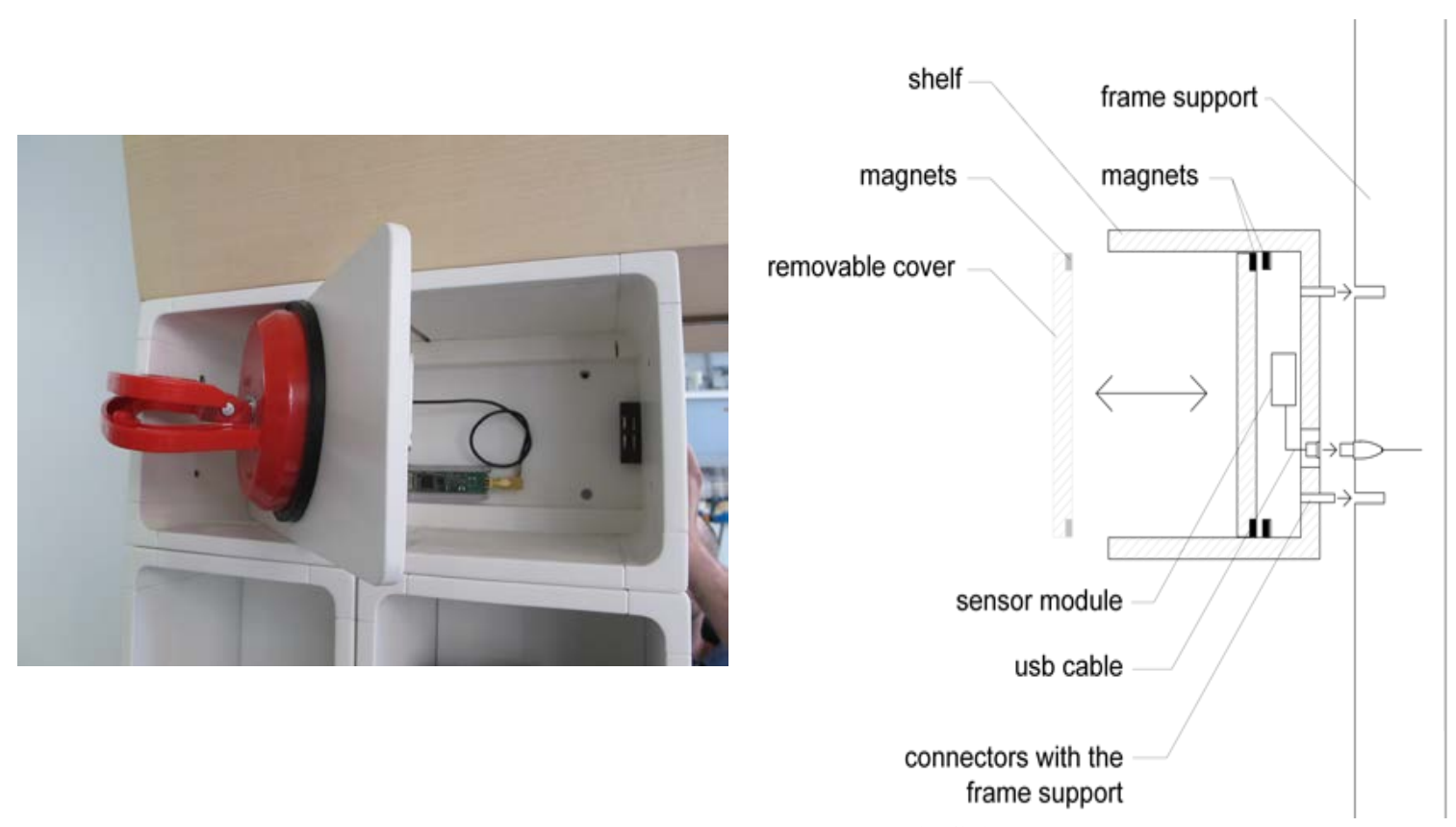

(d) Modular sensor infill
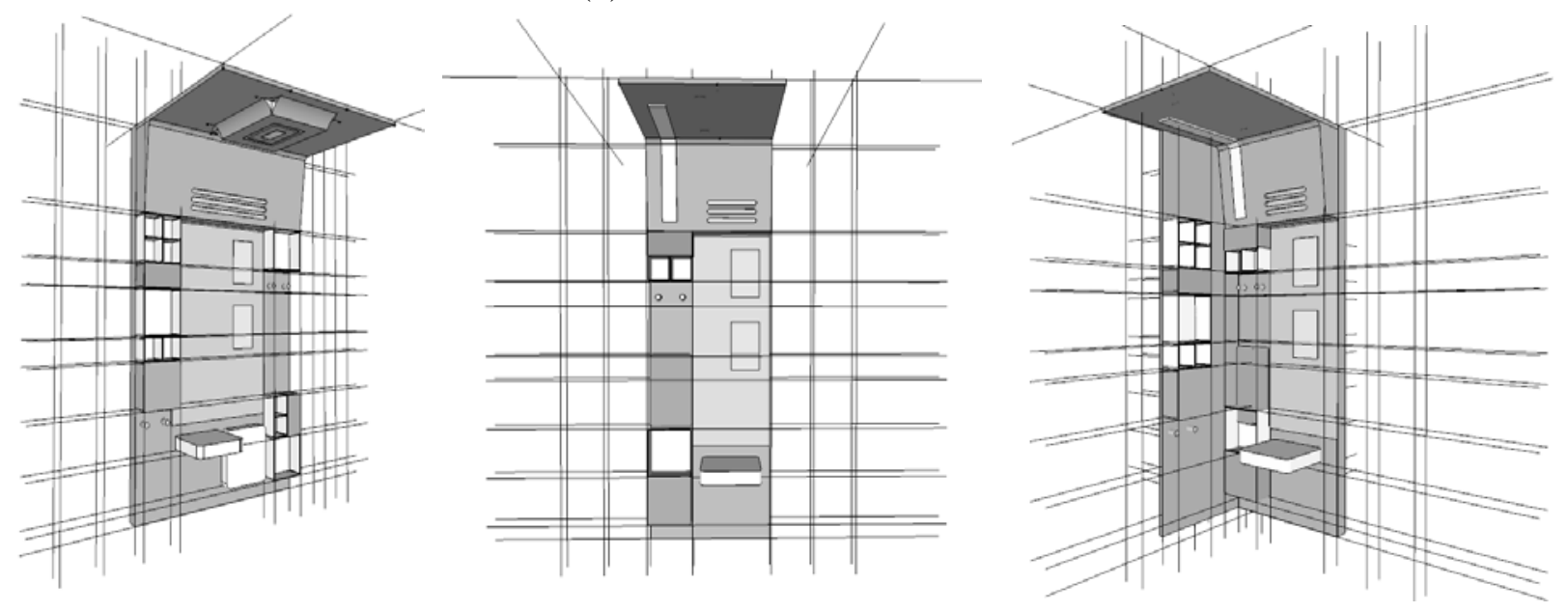

(e) Various configurations of the entrance RmR (Drawing on basis of MM Design)

Fig.12. User adaptation due to offered customization and modularity

\section{Results}

As mentioned in the introduction, the aim of the project was to address a lack of knowledge and guidelines for utilization of robotic rooms as practical and novel elements of future gerontechnology. Therefore, aa terminal-like system for the realization of intelligent, mechatronicbased and robotized environments, assisting elderly people in performing ADLs was developed, and tested and optimized. This was done in a systematic and scientific manner in four successive developmental phases. The following research findings were generated:

1. Contrary to initial assumptions, a robotic environment should only focus on strategically important parts of an apartment, on the user acceptance and on a cost-effective implementation.

2. Due to the RmR, structured environments limited to spatial sections can be created, which form the basis for the optimization of processes and the interaction of robotic sub-systems.

3. Built-in functions that serve the user (e.g. RFID-based reminders) can be used simultaneously to 
structure the environment and to provide information about objects and their position for other sub-systems.

4. The users require well-being, security and multimodality in an assistive environment in addition to the actual functional assistance cover (which should always be multidimensional).

5. The users can easier accept the use of a RmR if it is modular, and can be adapted to different life situations. It is important that users can purchase a cost-effective basic element, which can later be equipped, if desired, with other, more complex assistive functions and subsystems.

6. Apart from the users, the industry also prefers RmRs be incorporated with minimal intervention into the building and the shortest possible construction time without interference from conversion measures - as shown in development phase 4.

7. Following the evaluation in Development Phase 3, an open discussion with the test subjects revealed that innovative and intuitive gesture-driven or speech-driven user interfaces are more appropriate for elderly individuals.

8. The applied research method showed that a step by step approach in the development phases, a continuous involvement of users as well as a reduction in complexity, and the focus to a specific area of the environment (living environments are characterized as particularly highly complex by the variety of activities that take place), are necessary to introduce such a high-tech approach with user acceptance and marketability features.

Several functional mock-ups and prototypes were built, tested and evaluated for the selected Life Center (entrance). At the end of the project, a prototype was installed in a real home environment to undergo a long-term testing phase. This formed the basis for a set of modules and approaches that can now be utilized in further development of RmRs for the remaining Life Centers of the home environment.

\section{Construction Automation: Challenges and Solutions}

The proposed solution not only serves the use case of ageing and independent living, but also can be seen as a novel strategy to mass produce (or better: mass customize) and integrate smart home functions' required costs, time and resources efficiently into existing and also newly constructed buildings. The integration of smart home functions (and related advanced assistive functions and robotic systems - as outlined in this paper- as well as other smart functions, for example, those necessary to allow for the operational energy efficiency of buildings) into buildings in large-scale and at reasonable costs represents a major challenge for construction industry. Costs for smart or assistive buildings are significantly higher than for conventional buildings, which prevent its marketability. The automotive industry has already successfully addressed this 
challenge: it has been continuously optimizing the production process for decades, and thus has steadily reduced production costs. The savings are re-invested into research, development, increasing technology and optimization of the production process. This enables the companies to offer ever better-featured cars with relatively constant prices (for more information see [46]). The presented approach allows concentrating smart and assistive functions on a compact element that is, in terms of design of the building, modularily decoupled from the actual building and thus ready for factory-driven, automated prefabrication that can serve as the basis for reduction of production as well as installation costs of such systems. In this context, it must be concluded that advances in the field of construction automation (or respectively construction robotics, logistics, design of modular buildings, open buildings, etc.) are key for the large-scale diffusion of smart, assistive buildings. The opposite is also true: smart, assistive buildings can be seen as a driver for construction automation. Following Milgrom \& Robert's theory of complementarities [47], it can be concluded that both aspects, along with novel business and service models, are highly interdependent.

\section{Conclusion and Further Development}

In this article, the need for novel, robotics-based gerontechnolgy solutions, i.e. assistive, robotic rooms and RmRs was presented based on increasing elderly population numbers in leading industrial nations [48]. The research question and a classification of the approach in the broader context of research were also presented. The systemic, methodical, and structured development (four phases) approach, while strongly considering user feedback throughout the development was conducted. After the design of the overall system was described, a "Terminal” approach (cockpit systematic) partially structuring the environment, offering multidimensional assistance and a method for the systematic transfer of ADLs in assistance functions was formed. Furthermore, the overall system operation and the technical details of the assistive functions were presented. It has been shown that the system is built in an open and modular fashion so that more assistive functions (also provided by suppliers) can be easily integrated in the future.

The already embedded subsystems comprise of an intelligent ambient lighting system, a mobile robotic rover (Turtlebot) as an intuitive user-machine interface, an air quality purifying system, a standard touchpad interface (tablet PC) for real-time display of navigation and weather information, a reminder-alerting RFID-based monitoring system, a user vital signs display and measurement system, a shoes put-on/take-off assisting system, and an assistive standing-up/sittingdown robotic mechanism. Additionally, the methodical procedure in the individual phases of development were shown, and after several development cycles, the system gradually presented enhanced user acceptance and marketability due to the integration of user feedback throughout each 
development phase. The ultimate user acceptance has certainly not yet been achieved, but other necessary steps for commercialization are now clearly evident via the proposed system, which allows cost-efficient industrial manufacturing of RmRs. Furthermore, it has been shown how the system is designed to enable a higher-level product-service system. Finally, the results of the research project clearly indicate the potential for further development and scaling of the proposed approach in order to develop a series of RmRs, for all 6 identified home environment Life Centers.

In summary, this article describes the development of an innovative RmR that works towards autonomous and independent performance of ADLs in the home environment. The RmR combines technologies of e-Health, mobile robotics, machine-vision, sensor fusion, human-machine communication, etc., that provide elderly people with self-determined and independent living. In addition to numerous experimental tests and practical experience, a real-life apartment-based longterm study has been conducted in Italy. As the results showed that this is a promising approach and the $\mathrm{RmR}$, due to its modularity, is suitable for mass production, the current approach is extended into further RmR terminals (shown also in Figure 3b) in a follow-up project ("LISA Habitec").

In future projects, a stronger focus on the prevention aspect and the consideration for enhanced e-Health should be given. Different biosensor devices could be unobtrusively integrated into the home environment, i.e. ECG, pulse oximetry, body temperature measurement, etc. Telemedicine approaches should also be pursued in the future, in order for hospitals and clinics to experience patient crowding relief. Specifically during annual flu season (fall), the risk of an influenza virus for the elderly is not only higher, but also more dangerous [49]. Micro-robot rooms would also be desirable from a medical perspective. In addition to the assistance with the ADLs, the micro-robot rooms could also be the shifted "instrument" of GPs in the living environment.

Moreover, the authors introduce the follow-up project, “LISA Habitec”, a novel humanmachine interface that offers intuitive and adaptive manipulation in ADLs, using the Leap Motion controller and the Jaco arm [50]. Some researchers have already evaluated the efficiency of robotic systems, specifically robotic arms, used by disabled individuals in performing ADLs [51, 52]. Most manipulators are operated via a keyboard or joystick, which according to the complexity of the robotic arm, require a series of configurations and mode selection routines by pressing a series of buttons, in order to select the desired operating mode or to perform a specific trajectory path translation. The authors are conducting research on new ways to operate such devices in order to reduce the involved operation complexity as elderly people tend to face difficulties upon operating a complex interface, or refrain from using on a daily basis because they consider the interrelated controlling scheme complicated. Instead of using the original joystick "Kinova Joystick" of the Jaco arm and having to switch between different modes of control, the joystick is replaced with the Leap Motion controller that allows for an intuitive human-machine interface realization. The Leap 
Motion controller monitors the user's hand/hands, fingers, and all the accompanied positions and angles. All information regarding the user palm Cartesian position is retrieved from the controller and fed to the algorithm. The algorithm uses the current and previous information supplied by the controller and achieves an optimum realistic mapping between the user's real arm and the Jaco arm. The Leap Motion -Jaco arm interface system was adapted into the RmR (Figure 13).

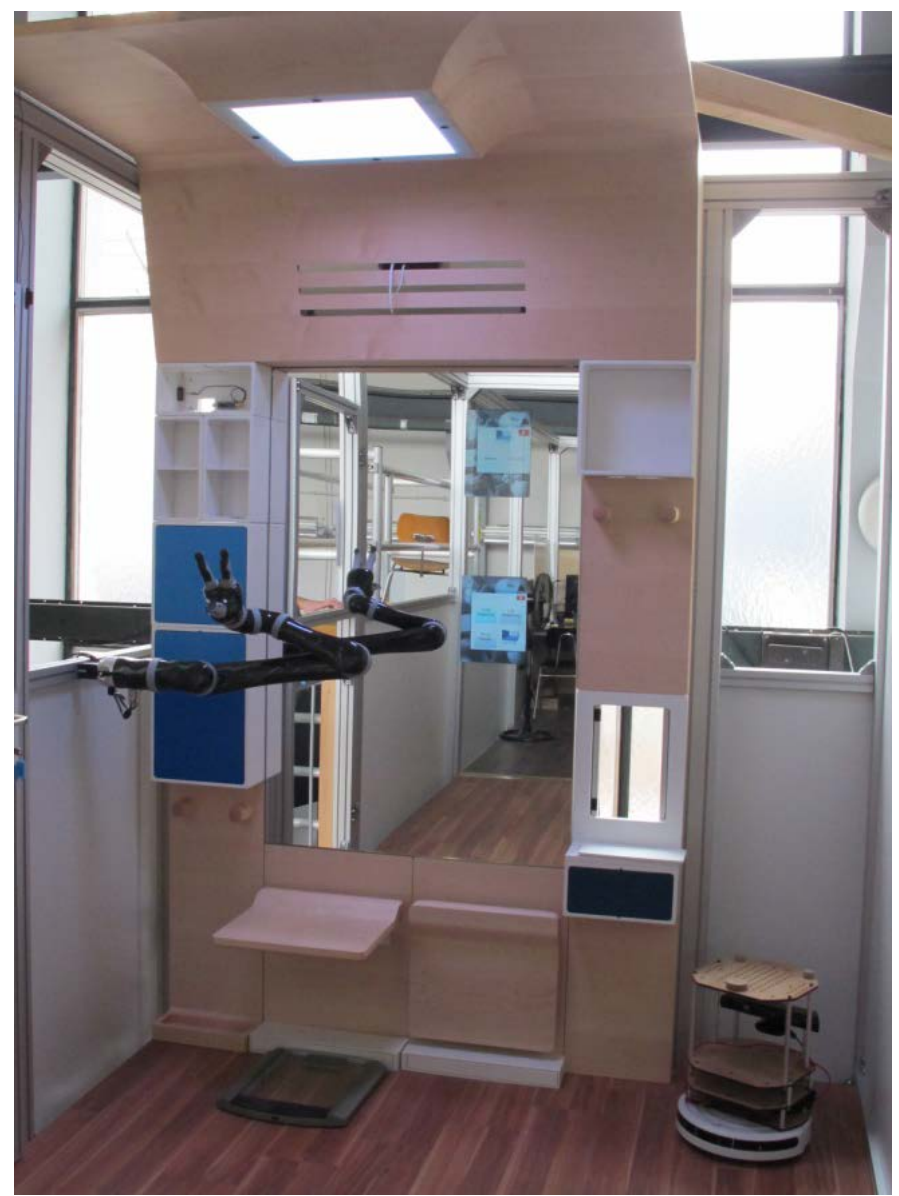

Fig.13. Intuitive manipulation within the RmR

The main contribution of developing such interfaces is to introduce the assistive services and functions that the Jaco arm offers in performing ADLs. Ageing society faces numerous challenges in performing simple tasks in ADLs. Nowadays, the caring of elderly people becomes more and more important. Individuals with upper limb impairments also face difficulties to perform ADLs, especially in cases where the impairments have resulted from spinal cord injuries, neuromuscular diseases, etc. Many technical aids have been developed to assist in impairments in the home environment. However, these assistive devices provide limited functionality and cannot address independence and autonomy in an efficient way [53, 54]. By introducing the Jaco arm within the proposed interactive home environment, and by focusing on assisting the user with various daily living activities through an intuitive, easy to operate, gesture driven compact size sensor, a new human-machine communication scheme can be established. 


\section{Acknowledgements}

The 2-year research project LISA (Living Independently in South Tyrol Aldo-Adige) was funded by the Province of Bolzano, with a total budget of approximately 1.2 million $€$. Apart from the Technical University of Munich, which formed the scientific backbone of the consortium, the following institutions and companies were involved in the realization: MM Design, Center Alpine Building / Frener \& Reifer, TIS Innovation Park, Pfeiffer Architects, Barth KG interior finishing. Evaluation in Development Phase 1 was conducted in collaboration with the Universita Degli Studi Di Trento and evaluation in Development Phase 3 was supported by Prof. Dr. K. Wessig (LudwigMaximilian University).

\section{References}

[1] Christensen K, Doblhammer G, Rau R, Vaupel JW, Ageing populations: the challenges ahead, The Lancet, 374 (9696) (2009) 1196-1208.

[2] Intille S, Larson K, Tapia EM, Designing and evaluating technology for independent aging in the home, Proceedings of the International. Conference on Aging, Disability and Independence, 2003.

[3] Wiener JM, Hanley RJ, Clark R, Van Nostra JF, Measuring the activities of daily living: comparisons across national surveys, Journal of Gerontology, Social Sciences, 46 (1990) 229-237.

[4] Krueger D, Ludwig A, On the Consequences of Demographic Change for Rates of Returns to Capital, and the Distribution of Wealth and Welfare. Journal of Monetary Economics, 54 (1) (2007) 49-87.

[5] Börsch-Supan A, Mariuzzo F, Our sample: 50+ in Europe. In: A. Börsch-Supan et al. Health, Ageing and Retirement in Europe - First Results from the Survey of Health, Ageing and Retirement in Europe, (2005) 30-34.

[6] Bähr M; et al, PASSAge - Personalisierte Mobilität, Assistenz und Service Systeme in einer alternden Gesellschaft, Proceedings of the $6^{\text {th }}$ German AAL-Conference, Berlin: VDI/VDE, 2012.

[7] Erdt S, Linner T, Herdener L, Rieß J, Roalter L, Schulz T, Struck M, Setz W, Bock T, Kranz M, Velioglu V, Moritz E, Systematic development of a complex personal health assistance system explained by the example of GEWOS activity furniture, Proceedings of the $5^{\text {th }}$ German AAL-Conference, Berlin: VDI/VDE, 2012.

[8] Maier H, Gampe J, Vaupel JW, Robine JM, (Eds.), Supercentenarians, Demographic Research Monographs, Heidelberg, Springer, (2010).

[9] Keupp H, Development towards a super-egocentric Society, Lecture on Munich Science Days on Demographic Change, November, 2013, Munich, 2013, lectures available in full length here, On-line: http://www.muenchnerwissenschaftstage.de/2013/download/download.html, Accessed: 13/01/2014.

[10] Sato T, Harada T, Mori T, Environment-type robot system "RoboticRoom” featured by behavior media, behavior contents, and behavior adaption, IEEE Transactions on Mechatronics, 9 (3) (2004) 529-534.

[11] Waseda Univ., Wabot House, On-line: http://www.wabot-house.waseda.ac.jp/html/e-top.htm, Accessed: 25.09.2013.

[12] Murakami K, Hasegawa T, KarazumeR, Kimuro Y, A Structured Environment with Sensor Networks for Intelligent Robots, Proceedings of IEEE Sensors, 26-29 October, Lecce, Italy, pp. 705-708, 2008

[13] Linner T, Kranz M, Roalter L, Bock T, Robotic and Ubiquitous Technologies for Welfare Habitat, Journal of Habitat Engineering, 3 (1) (2011) 101-111.

[14] Williams BC, Nayak PP, Immobile Robots: AI in the New Millenium. AI Magazine, Vol 17(3) (1996) 16-35.

[15] Shimizu N, A House of Sustainability: PAPI: Intelligent House in the Age of Ubiquitous Computing. Journal of Architecture and Urbanism (AU), Special Issue, 2005.

[16] Kidd CD, Orr R, Abowd GD, Atkeson CG, Essa IA, MacIntyre B, Mynatt ED, Starner T, Newstetter W, The Aware Home: A Living Laboratory for Ubiquitous Computing Research, Proceedings of the $2^{\text {nd }}$ International Workshop on Cooperative Buildings, Integrating Information, Organization, and Architecture, pp. 191-198, 1999. 
[17] Initlle S, Larson K, Tapia EM, Beaudin J, Kaushik P, Nawyn J, Rockinson R, Using a Live-in Laboratory for Ubiquitous Computing Research, Proceeding of the $4^{\text {th }}$ International Conference on Pervasive Computing, Dublin, Ireland, pp. 349-365, 2006.

[18] Larson K, Stephen I, MIT Open Source Building Alliance - A house_n initiative. Position Paper, MIT House_n, 2005.

[19] Haus der Gegenwart, Munich, Germany. Partners: Microsoft, BMW, Munich City. On-line: http://www.hausder-gegenwart.de/partner/, Accessed: 12/11/2009.

[20] Intelligent Networking: T-Com House. Siemens, Telecom Laboratories. Berlin, 2005-2006.

[21] Linner T, Bock T, Demographic Change Robotics: Mechatronic Assisted Living and Integrated Robot Technology, Introduction to Modern Robotics II, McDowall N, Chugo D, Yokota S (Eds.), ISBN 978-0980733075, (2012).

[22] Linner T, Kranz M, Roalter L, Bock T, Compacted and Industrially Customizable Ambient Intelligent Service Units: Typology, Examples and Performance, Proceedings of the $6^{\text {th }}$ International Conference on Intelligent Environments, 19-21 July, Kuala Lumpur, Malaysia, pp. 295-300, 2010.

[23] Meyer S, Rakotonirainy A, A Survey of Research on Context-Aware Homes, Proceedings of the Australasian information security workshop conference on ACSW frontiers, Adelaide, Australia, 21, pp. 159-169, 2003.

[24] Valli A, Natural Interaction White Paper, On-line: http://www.naturalinteraction.org/images/whitepaper.pdf, (2007).

[25] Habraken NJ, The Structure of the Ordinary - Form and Control in the Built Environment. Boston: The MIT Press, (2000).

[26] Kendall S, Teicher J, Residential Open Building. E\&FN SPON, (2010).

[27] Linner T, Bock T, Evolution of large-scale Industrialization and Service: Innovation in Japanese Prefabrication Industry, Journal of Construction Innovation: Information, Process, Management, 12 (2) (2012) 156-178.

[28] Bock T, Linner T, Mass Customization und Plattform basierte, adaptive Baukastensysteme für “Ambient Assisted Living”-Umgebungen, Proceedings of the $3^{\text {rd }}$ Ambient Assisted Living Conference, Berlin. Germany, 2010.

[29] Bock T, A study on Robot-Oriented Construction and Building System, Thesis for Doctorate of Engineering, University of Tokyo, Report Number. 108066, 1989.

[30] Linner T, Eibisch N, Bock T, Strategy for the development of assistive Environments based on geriatric Assessment, Proceedings of 28th International Symposium on Automation and Robotics in Construction (ISARC), Seoul, Korea, 2011.

[31] Freund H, Geriatrisches Assessment und Testverfahren: Grundbegriffe, Anleitungen, Behandlungspfade, Stuttgart: Kohlhammer, (2009).

[32] McDowell I, Newell C, Measuring Health: A Guide to Rating Scales and Questionnaires, 2nd edition, New York: Oxford University Press, 1996.

[33] Bookman A, Harrington M, Pass L, Reisner E, Family Caregiver Handbook, Cambridge, MA: Massachusetts Institute of Technology, 2007.

[34] Georgoulas C, Linner T, Kasatkin A, Bock T, An AmI Environment Implementation: Embedding TurtleBot into a novel Robotic Service Wall, Proceedings of the $7^{\text {th }}$ German Conference on Robotics (ROBOTIK), Munich, Germany, May 2012, pp. 117-122, 2012.

[35] Dissanayake MWMG, Newman P, Clark S, Durrant-Whyte HF, Csorba M, A solution to the simultaneous localization and map building (SLAM) problem, Robotics and Automation, IEEE Transactions on, 17 (3) (2001) 229241.

[36] Microsoft Corp. Redmond WA. Kinect Xbox 360.

[37] Murray D, Little JJ, Using real-time stereo vision for mobile robot navigation, Journal of Autonomous Robots,8 (2) (2000) 161-171.

[38] Finkelzeller K, The RFID Handbook, 2nd ed., John Wiley \& Sons, 2003.

[39] Want R, An introduction to RFID technology, IEEE Pervasive Computing, 5(1) (2006) 25-33.

[40] Biocomfort Diagnostics, On-line: http://www.biocomfort.de/health-manager-platform-283.html, Accessed: 12/02/2014.

[41] Georgoulas C, Linner T, Bock T, Towards a Vision Controlled Robotic Home Environment, Journal of Automation in Construction, ISSN: 0926-5805, doi: http://dx.doi.org/10.1016/j.autcon.2013.06.010, Elsevier, (2014) 106-116.

[42] Sterbefälle insgesamt 2011 nach den 10 häufigsten Todesursachen der ICD-10; On-line: https://www.destatis.de/DE/ZahlenFakten/GesellschaftStaat/Gesundheit/Todesursachen/Tabellen/SterbefaelleIns gesamt.html, Accessed: 12/11/2013.

[43] Meyer J, Bockisch A, Darius H, Heusch G, Hort W, Mohr-Kahaly S, Rupprecht HJ, Voigtländer T, Koronare Herzkrankheit und Herzinfarkt, Springer Berlin Heidelberg, pp. 27-142, (2006). 
[44] LISA Project Evaluation On-line: http://www.br2.ar.tum.de/Download/Lisa-Funktionsanalyse.zip, Accessed: $14 / 02 / 2014$.

[45] Jähn K, Nagel E, e-Health, Berlin: Springer, (2004).

[46] Linner T, Automated and Robotic Construction: Integrated Automated Construction Sites, Dr.-Ing. Dissertation, Technische Universität München, (2013)

[47] Milgrom P, Roberts J, The Economics of Modern Manufacturing: Technology, Strategy and Organization, American Economic Review 80/3 (1990) pp. 511-528.

[48] Krueger, Dirk, Ludwig, Alexander, 2007. On the consequences of demographic change for rates of return to capital, and the distribution of wealth and welfare. Journal of Monetary Economics 54 (1), 49-87.

[49] Carrat F, Valleron AJ, Influenza mortality among the elderly in France', 1980-90: how many deaths may have been avoided through vaccination?, Journal of Epidemiology and Communication Health, 49 (1995) 419-425.

[50] Bassily D, Georgoulas C, Güttler J, Linner T, Bock T, Intuitive and Adaptive Robotic Arm Manipulation using the Leap Motion Controller, accepted for Oral Presentation in the joint conference of the 45th International Symposium on Robotics (ISR 2014) and the 8th German Conference on Robotics (ROBOTIK 2014), Munich, Germany, June 2, 2014.

[51] Romer G, Stuyt HJA, Peters A, Cost-savings and economic benefits due to the assistive robotic manipulator (ARM). Proceedings of the 9th International Conference on Rehabilitation Robotics (ICORR), Chicago, IL, pp. 201-204, 2005.

[52] Stanger CA, Devices for assisting manipulation: a summary of user task priorities, IEEE Transactions on Rehabilitation Engineering, 2 (4) (1994) 256-265.

[53] Atkins MS, Baumgarten JM, Yasuda YL, Adkins R, Waters RL, Leung P, Requejo P, Mobile arm supports: evidence based benefits and criteria for use, Journal of Spinal Cord Medicine, 31 (4) (2008) 388-393.

[54] Garber SL, Gregorio TL, Upper extremity assistive devices: assessment of use by spinal cord injured patients with quadriplegia, American Journal of Occupational Therapy, 44 (2) (1990) 126-131. 